\title{
Electric Dipole Moments of Light Nuclei From Chiral Effective Field Theory
}

\author{
J. de Vries ${ }^{1}$, R. Higa ${ }^{1,2}$, C.-P. Liu ${ }^{3}$, E. Mereghetti ${ }^{4,5}$, I. Stetcu ${ }^{6}$, \\ R. G. E. Timmermans ${ }^{1}$, and U. van Kolck ${ }^{4}$ \\ ${ }^{1}$ KVI, Theory Group, University of Groningen, \\ 9747 AA Groningen, The Netherlands \\ ${ }^{2}$ Instituto de Física, Universidade de São Paulo, \\ C.P. 66318, 05389-970 São Paulo, SP, Brazil \\ ${ }^{3}$ Department of Physics, National Dong Hwa University, \\ Shoufeng, Hualien 97401, Taiwan \\ ${ }^{4}$ Department of Physics, University of Arizona, \\ Tucson, AZ 85721, USA \\ ${ }^{5}$ Ernest Orlando Lawrence Berkeley National Laboratory, \\ University of California, Berkeley, CA 94720, USA \\ ${ }^{6}$ Department of Physics, University of Washington, Box 351560, \\ Seattle, WA 98195-1560, USA
}

\begin{abstract}
We set up the framework for the calculation of electric dipole moments (EDMs) of light nuclei using the systematic expansion provided by chiral effective field theory (EFT). We take into account parity $(P)$ and time-reversal $(T)$ violation which, at the quark-gluon level, originates from the QCD vacuum angle and dimension-six operators capturing physics beyond the Standard Model. We argue that EDMs of light nuclei can be expressed in terms of six low-energy constants that appear in the $P$ - and $T$-violating nuclear potential and electric current. As examples, we calculate the EDMs of the deuteron, the triton, and ${ }^{3} \mathrm{He}$ in leading order in the EFT expansion.
\end{abstract}




\section{Introduction}

Permanent electric dipole moments (EDMs) [1, 2] of particles, nuclei, atoms, and molecules provide stringent bounds on sources of parity $(P)$ and time-reversal $(T)$ violation beyond the phase of the quark-mixing matrix [3] in the Standard Model (SM). Experiments in preparation [4] aim to improve the current bound on the neutron EDM, $\left|d_{n}\right|<2.9 \cdot 10^{-26} e$ $\mathrm{cm}$ [5], by up to two orders of magnitude. At the same time, proposals [6] exist for the measurement of EDMs of charged particles in storage rings, in particular the proton for which an indirect bound, $\left|d_{p}\right|<7.9 \cdot 10^{-25} \mathrm{e} \mathrm{cm}$, has been extracted from the ${ }^{199} \mathrm{Hg}$ EDM [7] — and the deuteron. It may be possible, moreover, to measure in this way the EDMs of other light nuclei, for example ${ }^{3} \mathrm{He}$ and ${ }^{3} \mathrm{H}$, as well. A nonzero EDM signal in this new generation of experiments would be an unambiguous sign of new physics at energy scales similar to those probed by the LHC, since the EDMs resulting from the quark-mixing matrix [8] are orders of magnitude below the current experimental limits.

An important outstanding question is whether it will be possible to identify from the experimental results the microscopic parity- and time-reversal-violating (PP) source. The SM contains, apart from the quark-mixing phase and its lepton analog, also the PF QCD $\bar{\theta}$ term [9]. This interaction has dimension four and one might expect it to give the main contribution to hadronic PT. However, since the experimental upper limit on the neutron EDM constrains the vacuum angle $\bar{\theta}$ to be unnaturally small [10, 11], $\bar{\theta} \lesssim 10^{-10}$, nonSM contributions from higher-dimensional PT sources can be relevant, or even dominant. These higher-dimensional operators originate from an ultraviolet complete theory at a high energy scale $M_{7}$ beyond the electroweak scale. The first such effective PP operators one can write down have effective dimension six [12, 13, 14, 15], viz. the quark EDM (qEDM), the quark and gluon chromo-electric dipole moments (qCEDM and gCEDM, respectively), and two four-quark (FQ) interactions.

It is difficult to calculate hadronic properties directly from a Lagrangian written in terms of quark and gluon fields. Still, long-distance effects of the strong interactions can be described independently of assumptions about the dynamics of QCD if one uses the low-energy effective field theory (EFT) known as chiral perturbation theory $(\chi \mathrm{PT})$ [16, 17] (for reviews see Refs. [18, 19]), which translates microscopic operators into operators that contain nucleon, pion, and photon fields. After the translation, one is able to calculate hadronic properties directly from the effective Lagrangian. For the nucleon EDM (and its associated form factor) stemming from the dimension-four $\bar{\theta}$ term, this method was first used in Refs. [10, 11], and later extended in the context of $S U(2) \times S U(2)$ [20, 21, 22, 23] and $S U(3) \times S U(3)[24,25] \chi \mathrm{PT}$. In this way it is possible to establish a link to $P T$ conserving $(P T)$ interactions [21, 22, 23].

This approach can be generalized to include the dimension-six sources [26, 23, 27]. Different PF sources at the quark-gluon level produce different hadronic interactions. The effective chiral Lagrangian includes not only interactions that stem from spontaneous chiral symmetry breaking and are therefore chiral invariant, but also interactions that break chiral symmetry in the same way as chiral-symmetry-breaking operators at the QCD level. Thus, while they all break $P$ and $T$, the dimension-six operators break chiral 
symmetry differently from each other and from the $\bar{\theta}$ term. Given enough observables it should be possible to separate the various PT sources on the basis of the hadronic interactions that they generate.

Recently it was argued [28 that a measurement of the deuteron EDM in combination with the neutron or proton EDM could partially separate the fundamental PT sources. A measurement of the deuteron EDM significantly larger than the nucleon EDM would point toward new physics in the guise of a quark chromo-EDM. The calculation was based on a perturbative-pion approach [29, 30] to nuclear EFT, which assumes that pion exchange can be treated in perturbation theory. A major advantage of this approach is that analytical results can be obtained. On the other hand, such a framework is applicable only below the momentum scale $(\sim 300 \mathrm{MeV})$ at which one-pion-exchange (OPE) becomes significant. This is the case for nuclei where the binding momentum per nucleon is small compared to the pion mass, but even then the size of uncertainties is set by the inverse of the relatively low energy scale.

Our goal in this article is to provide a framework for the calculation of the EDMs of light nuclei using chiral EFT with nonperturbative OPE [31, 32, 33, 30]. By treating OPE nonperturbatively, the EFT gets extended to higher momenta and thus denser nuclei, and convergence improves. The fact that nuclear binding momenta are small in the typical scale of QCD $(\sim 1 \mathrm{GeV})$ is sufficient for a general power counting that is able to estimate which hadronic interactions are dominant for each fundamental PT source. The PPT potential has been derived previously [34], and here we obtain the associated PP currents. As explicit examples we consider the EDMs of the deuteron $\left({ }^{2} \mathrm{H}\right)$, the triton $\left({ }^{3} \mathrm{H}\right)$, and the helion $\left({ }^{3} \mathrm{He}\right){ }^{1}$.

The EDMs of the deuteron [36, 37, 38, 39, 40] and helion [41, 42] have been investigated previously within traditional meson-exchange frameworks. In the most comprehensive studies [39, 42] one started from "realistic" nuclear-force models and a general PT nucleon-nucleon $(N N)$ interaction [39, 43]. The EDMs were expressed in terms of three PT non-derivative pion-nucleon interactions, which are often assumed to be of similar size and dominate the EDMs, and in addition short-range PT interactions due to the exchange of heavier mesons were included. The major advantage of a chiral EFT framework is that it has a direct link to QCD and exploits the chiral properties of the fundamental PF sources. Moreover, the power-counting scheme allows a perturbative expansion such that the theoretical uncertainties can be estimated and the results can be improved systematically.

When the chiral-symmetry properties of the dimension-four and dimension-six operators are considered new insights are in fact obtained [21, 22, 26, 23, 28, 27]. At leading order, only two of the three PT pion-nucleon interactions contribute. Moreover, there are in general at the same order more contributions, viz. short-range contributions to the neutron and proton EDMs and two PPT $N N$ contact interactions. As we will demonstrate below, the EDMs of light nuclei can be expressed in terms of these six PT parameters, or low-energy constants (LECs). (Other PP moments, such as the deuteron magnetic

\footnotetext{
${ }^{1}$ Similar calculations are being carried out by a group at FZ Jülich 35 .
} 
quadrupole moment, depend in addition on PT pion-nucleon-photon interactions 28].) For three of the four PT sources, only a subset of these six LECs is in fact needed. Each LEC can in principle be calculated from the underlying PT source using an explicit solution of QCD at low energies, for example through lattice simulations. Compared to nucleons, the EDMs of light nuclei can give crucial complementary information about the fundamental PP source. However, the conventional assumption that the three PP pion-nucleon interactions can cover the whole range of nuclear EDMs is oversimplified.

For the PT potential we use here realistic phenomenological potentials [44, 45, 46]. This "hybrid" approach [47] is justified whenever there is little sensitivity to the details of short-range physics, since such realistic potentials all include the long-range pion exchange that appears in chiral EFT at LO. Such an approach has been tested successfully for other observables [30], such as the PT form factors of the deuteron [48] and PPT NN observables [49. The results in Refs. [39, 42] suggest that the same is true for EDMs, and we partially confirm this below. We use the codes from Refs. [39, 42, but we recast and extend the results in the framework of chiral EFT with nonperturbative OPE. In particular, we apply power counting in order to make more model-independent statements. The cases of the helion and the triton are typical of a generic nucleus. However, in the deuteron, because of its isoscalar character, the formally LO contribution from the $\bar{\theta}$ term vanishes [38, 39, 28], a property expected [50] for nuclei with equal number of protons and neutrons, $N=Z$. We exploit the systematic character of EFT to extend the deuteron calculation for the $\bar{\theta}$ term to the first non-vanishing order.

Our article is organized as follows. In Section 2, we present the $P$ - and $T$-conserving and violating interactions relevant for the calculation of light nuclear EDMs. In Section 3 we discuss in general the power counting of the various contributions, and present the leading PT potentials and currents, while in Section 4 we specifically address nuclei with $N=Z$. Next, we evaluate the EDM of the deuteron in Section 5 and the EDMs of the helion and the triton in Section 6. In Section 7 we discuss our results and their implications. Appendices are devoted to the expression of potential and currents in coordinate space.

\section{Chiral Perturbation Theory}

$\chi \mathrm{PT}$ is the EFT of QCD for processes involving momenta $Q \sim m_{\pi} \ll M_{\mathrm{QCD}}$, where $m_{\pi}$ is the pion mass and $M_{\mathrm{QCD}} \sim 1 \mathrm{GeV}$ is the characteristic scale of QCD. At such momenta the relevant degrees of freedom are nucleons, photons, and pions. The (approximate) chiral symmetry of QCD, $S U_{L}(2) \times S U_{R}(2) \sim S O(4)$, plays a very important role, because it constrains the form of the interactions involving the (pseudo) Goldstone bosons associated with its spontaneous breaking, the pions. In this section we review these interactions, in both PT and PT sectors of the theory.

The $\chi \mathrm{PT}$ Lagrangian contains all interactions allowed by the symmetries of QCD. Each interaction is written in terms of pion $(\boldsymbol{\pi})$, nucleon $(N)$, and photon $\left(A_{\mu}\right)$ fields. The constraints of (global) chiral and (gauge) electromagnetic symmetries can be incorporated 
through the use of covariant derivatives,

$$
\left(D_{\mu} \boldsymbol{\pi}\right)_{a}=\frac{1}{D}\left(\partial_{\mu} \delta_{a b}+e A_{\mu} \varepsilon_{3 a b}\right) \pi_{b}
$$

for the pion,

$$
\mathcal{D}_{\mu} N=\left[\partial_{\mu}+\frac{i}{F_{\pi}^{2}} \boldsymbol{\tau} \cdot\left(\boldsymbol{\pi} \times D_{\mu} \boldsymbol{\pi}\right)+i e A_{\mu} \frac{1+\tau_{3}}{2}\right] N
$$

for the nucleon, and

$$
F_{\mu \nu}=\partial_{\mu} A_{\nu}-\partial_{\nu} A_{\mu}
$$

for the photon. Here $F_{\pi} \simeq 185 \mathrm{MeV}$ is the pion decay constant,

$$
D \equiv 1+\frac{\pi^{2}}{F_{\pi}^{2}}
$$

$\boldsymbol{\tau}$ are the Pauli matrices in isospin space, and $e$ is the proton electric charge. Since $m_{N} \sim M_{\mathrm{QCD}}$, nucleons are approximately non-relativistic in the processes of interest, and Lorentz invariance is incorporated order by order in the EFT expansion [17]. We denote the (small) nucleon four-velocity by $v_{\mu}$ and its spin by $S_{\mu}$; in the nucleon rest frame, $v_{\mu}=(1, \overrightarrow{0})$ and $S^{\mu}=(0, \vec{\sigma} / 2)$ in terms of the Pauli matrices in spin space, $\vec{\sigma}$.

Chiral-invariant interactions are built with pion covariant derivatives, while explicit chiral symmetry breaking is introduced by the average quark mass $\bar{m}=\left(m_{u}+m_{d}\right) / 2$, by the quark mass difference $m_{d}-m_{u}=2 \bar{m} \varepsilon$, by electromagnetic interactions, and $\not P$ and/or TT interactions. Each interaction with the correct symmetry transformation properties has a strength determined by details of the QCD dynamics. Until they are known, they are estimated using naive dimensional analysis (NDA) [16, 51, 13]. For example, the pion mass term originates from explicit chiral-symmetry breaking by the average quark mass, so $m_{\pi}^{2}=\mathcal{O}\left(\bar{m} M_{\mathrm{QCD}}\right)$. The LECs of other chiral-breaking interactions proportional to powers of $\bar{m}$ and $\bar{m} \varepsilon$ can then be written in terms of $m_{\pi}^{2} / M_{\mathrm{QCD}}$. Exchange of hard photons leads to interactions among hadrons that are proportional to the fine-structure constant $\alpha_{\mathrm{em}}=e^{2} / 4 \pi$. For simplicity, we count $\varepsilon \sim 1 / 3$ as $\mathcal{O}(1)$ and $\alpha_{\mathrm{em}} / 4 \pi$ as $\mathcal{O}\left(m_{\pi}^{3} / M_{\mathrm{QCD}}^{3}\right)$, since numerically $\alpha_{\mathrm{em}} / 4 \pi \sim \varepsilon m_{\pi}^{3} /\left(2 \pi F_{\pi}\right)^{3}$. It is convenient to organize the infinity of effective interactions in the Lagrangian using an integer "chiral index" $\Delta$ and the number $f$ of fermion fields [16, 18]:

$$
\mathcal{L}=\sum_{\Delta=0}^{\infty} \sum_{f} \mathcal{L}_{f}^{(\Delta)}
$$

where $\Delta=d+f / 2-2 \geq 0$, with $d$ the number of covariant derivatives and powers of $m_{\pi}$. The index $\Delta$ tracks the number of powers of $M_{\mathrm{QCD}}^{-1}$.

\section{$2.1 \quad P$ - and $T$-conserving chiral Lagrangian}

The calculation of the PF potential and currents, which we need in order to evaluate nuclear EDMs, requires also PT interactions, which we summarize here. (A more complete 
list can be found in, for example, Refs. [19, 30, 52, 32, 53].) These interactions result from the quark (color-gauged) kinetic and mass terms in the QCD Lagrangian.

The terms we need in the PT Lagrangian are

$$
\begin{aligned}
\mathcal{L}_{f \leq 2, P T}^{(0,1,2)}= & \frac{1}{2} D_{\mu} \boldsymbol{\pi} \cdot D^{\mu} \boldsymbol{\pi}-\frac{m_{\pi}^{2}}{2 D} \boldsymbol{\pi}^{2}+\bar{N} i v \cdot \mathcal{D} N-\frac{1}{2 m_{N}} \bar{N}\left[\mathcal{D}^{2}-(v \cdot \mathcal{D})^{2}\right] N \\
& -\frac{\breve{\delta} m_{\pi}^{2}-\delta m_{N}^{2}}{2 D^{2}}\left(\boldsymbol{\pi}^{2}-\pi_{3}^{2}\right)-\frac{\delta m_{\pi}^{2}}{2 D^{2}} \pi_{3}^{2}-\left(\delta m_{N}+\breve{\delta} m_{N}\right)(\boldsymbol{\pi} \times v \cdot D \boldsymbol{\pi})_{3} \\
& -\frac{2 g_{A}}{F_{\pi}} D_{\mu} \boldsymbol{\pi} \cdot \bar{N} \boldsymbol{\tau} S^{\mu} N+\frac{\beta_{1}}{F_{\pi}}\left(D_{\mu} \pi_{3}-\frac{2 \pi_{3}}{F_{\pi}^{2} D} \boldsymbol{\pi} \cdot D_{\mu} \boldsymbol{\pi}\right) \bar{N} S^{\mu} N \\
& -\frac{g_{A} \delta m_{N}}{F_{\pi} m_{N}}\left[i \bar{N}(\boldsymbol{\tau} \times \boldsymbol{\pi})_{3} S \cdot \mathcal{D} N+\text { H.c. }\right] \\
& -\frac{e}{16 m_{N}^{2}} \varepsilon^{\alpha \beta \mu \nu} F_{\mu \nu}\left\{i \bar{N}\left[1+2 \kappa_{0}+\left(1+2 \kappa_{1}\right) \tau_{3}\right] S_{\alpha} \mathcal{D}_{\beta} N+\text { H.c. }\right\} .
\end{aligned}
$$

The pion kinetic and mass terms have $\Delta=0$. For notational simplicity, we choose to absorb in the pion mass $m_{\pi}$ a correction $\propto \bar{m}^{2}$. At $\Delta=1$, the leading electromagnetic contribution to the pion mass splitting appears, $\breve{\delta} m_{\pi}^{2}=\mathcal{O}\left(\alpha_{\mathrm{em}} M_{\mathrm{QCD}}^{2} / 4 \pi\right)$, while the quark-mass difference contribution, $\delta m_{\pi}^{2}=\mathcal{O}\left(\varepsilon^{2} m_{\pi}^{4} / M_{\mathrm{QCD}}^{2}\right)$, is smaller by one power of $\varepsilon m_{\pi} / M_{\mathrm{QCD}}$. The pion mass splitting, $m_{\pi^{ \pm}}^{2}-m_{\pi^{0}}^{2}=\breve{\delta} m_{\pi}^{2}-\delta m_{\pi}^{2}=(35.5 \mathrm{MeV})^{2}$ [54], is dominated by the electromagnetic contribution. The nucleon kinetic terms have $\Delta=0,1$. Again for simplicity, the average nucleon mass $m_{N}$ absorbs a correction $\propto \bar{m}$, the sigma term. Entering at $\Delta=1,2$, the nucleon mass splitting, $m_{n}-m_{p}=\delta m_{N}+\breve{\delta} m_{N}=1.29$ $\mathrm{MeV}$ [54] also receives contributions from electromagnetism and from the quark masses. In this case, the quark-mass contribution $\delta m_{N}$ is expected to be the largest. By dimensional analysis $\delta m_{N}=\mathcal{O}\left(\varepsilon m_{\pi}^{2} / M_{\mathrm{QCD}}\right)$, and lattice simulations estimate it to be $\delta m_{N}=2.26 \pm 0.57 \pm 0.42 \pm 0.10 \mathrm{MeV}$ [55], which is in agreement with an extraction from charge-symmetry breaking in the $p n \rightarrow d \pi^{0}$ reaction [56]. The electromagnetic contribution is $\breve{\delta} m_{N}=\mathcal{O}\left(\alpha_{\mathrm{em}} M_{\mathrm{QCD}} / 4 \pi\right)$, which is $\mathcal{O}\left(\varepsilon m_{\pi}^{3} / M_{\mathrm{QCD}}^{2}\right)$ and about the $20 \%$ of $\delta m_{N}$. From the Cottingham sum rule [57] one finds $\breve{\delta} m_{N}=-(0.76 \pm 0.30) \mathrm{MeV}$, which is consistent with dimensional analysis. In order to achieve the form (6) for $\mathcal{L}_{P T}$, we have used a field redefinition [53] to eliminate the nucleon mass difference term in favor of pionic mass and interaction terms. In this way, the nucleon mass to be used in nucleon propagators is simply $m_{N}$. The operator with LEC $g_{A}$ is the usual pion-nucleon axial coupling appearing at $\Delta=0$. We also absorb subleading corrections in it, so that the Goldberger-Treiman relation for the strong pion-nucleon constant, $g_{N N \pi}=2 g_{A} m_{N} / F_{\pi}$, applies without an explicit discrepancy. If for the pion-nucleon coupling constant we use $g_{N N \pi}=13.07$ [58], then $g_{A}=1.29$. Its isospin-violating counterpart is the operator with LEC $\beta_{1}=\mathcal{O}\left(\varepsilon m_{\pi}^{2} / M_{\mathrm{QCD}}^{2}\right)$ at $\Delta=2$. At present there are only bounds on $\beta_{1}$ from isospin violation in $N N$ scattering. The Nijmegen partial-wave analysis of $N N$ scattering data gives $\beta_{1}=(0 \pm 9) \cdot 10^{-3}$ [59], which is comparable to estimates of $\beta_{1}$ from $\pi-\eta$ mixing. At $\Delta=2$ there is another isospin-violating pion-nucleon interaction generated by nucleon recoil and the nucleon mass difference. Finally, also at $\Delta=2$ there is a relativistic 
correction [60] to the electromagnetic coupling of the nucleon involving the isoscalar and isovector components of the anomalous magnetic moment, respectively $\kappa_{0}=-0.12$ and $\kappa_{1}=3.7$.

\section{$2.2 \quad P$ - and $T$-violating chiral Lagrangian}

The lowest-dimension PF operator that can be added to the PT QCD Lagrangian is the dimension-four $\bar{\theta}$ term. With an appropriate choice of the quark fields $q=(u, d)^{T}$, the $\bar{\theta}$ term can be expressed as a complex mass term [10],

$$
\mathcal{L}_{P P}, \operatorname{dim}=4=m_{\star} \bar{\theta} \bar{q} i \gamma_{5} q
$$

where $m_{\star}=m_{u} m_{d} /\left(m_{u}+m_{d}\right)=\mathcal{O}\left(m_{\pi}^{2} / M_{\mathrm{QCD}}\right)$ and $\bar{\theta}$ is the QCD vacuum angle, here already assumed to be small, $\bar{\theta} \lesssim 10^{-10}$, as indicated by the experimental bound on the neutron EDM.

The smallness of $\bar{\theta}$ leaves room for other PP sources in the strong interactions, which have their origin in an ultraviolet-complete theory at a high energy scale, such as, for example, supersymmetric extensions of the Standard Model [15]. Well below the scale $M_{T}$ characteristic of $T$ violation, we expect PT effects to be captured by the lowestdimension interactions among Standard Model fields that respect $S U_{c}(3) \times S U_{L}(2) \times U_{Y}(1)$ gauge symmetry. Above $M_{\mathrm{QCD}}$, strong interactions are described by the most general Lagrangian with Lorentz, color, and electromagnetic gauge invariance among the lightest quarks, gluons, and photons. The effectively dimension-six PT terms at this scale can be written as [12, 13, 14, 15]

$$
\begin{aligned}
\mathcal{L}_{\not P F} \text { dim }=6= & -\frac{1}{2} \bar{q}\left(d_{0}+d_{3} \tau_{3}\right) \sigma^{\mu \nu} i \gamma^{5} q F_{\mu \nu}-\frac{1}{2} \bar{q}\left(\tilde{d}_{0}+\tilde{d}_{3} \tau_{3}\right) \sigma^{\mu \nu} i \gamma^{5} \lambda^{a} q G_{\mu \nu}^{a} \\
& +\frac{d_{W}}{6} \varepsilon^{\mu \nu \lambda \sigma} f^{a b c} G_{\mu \rho}^{a} G_{\nu}^{b \rho} G_{\lambda \sigma}^{c}+\frac{1}{4} \operatorname{Im} \Sigma_{1}\left(\bar{q} q \bar{q} i \gamma^{5} q-\bar{q} \boldsymbol{\tau} q \cdot \bar{q} \boldsymbol{\tau} i \gamma^{5} q\right) \\
& +\frac{1}{4} \operatorname{Im} \Sigma_{8}\left(\bar{q} \lambda^{a} q \bar{q} \lambda^{a} i \gamma^{5} q-\bar{q} \lambda^{a} \boldsymbol{\tau} q \cdot \bar{q} \lambda^{a} \boldsymbol{\tau} i \gamma^{5} q\right)
\end{aligned}
$$

in terms of the gluon field strength $G_{\mu \nu}^{a}$, the Gell-Mann matrices $\lambda^{a}$ in color space, and the associated structure constants $f^{a b c}$. In Eq. (8) the first (second) term represents the isoscalar $d_{0}\left(\tilde{d}_{0}\right)$ and isovector $d_{3}\left(\tilde{d}_{3}\right)$ components of the qEDM (qCEDM). Although these interactions have canonical dimension five, they originate just above the Standard Model scale $M_{W}$ from dimension-six operators [12] involving in addition the carrier of electroweak symmetry breaking, the Higgs field. They are thus proportional to the vacuum expectation value of the Higgs field, which can be traded in for the ratio of the quark mass to Yukawa coupling, $m_{q} / f_{q}$. Writing the proportionality constant as $e \delta_{q} f_{q} / M_{\mathcal{T}}^{2}\left(4 \pi \tilde{\delta}_{q} f_{q} / M_{\mathcal{T}}^{2}\right)$, we have

$$
d_{0,3} \sim \mathcal{O}\left(e \delta_{0,3} \frac{\bar{m}}{M_{T}^{2}}\right), \quad \tilde{d}_{0,3} \sim \mathcal{O}\left(4 \pi \tilde{\delta}_{0,3} \frac{\bar{m}}{M_{T}^{2}}\right)
$$


in terms of the average light-quark mass $\bar{m}$ and the dimensionless factors $\delta_{0,3}$ and $\tilde{\delta}_{0,3}$ that represent typical values of $\delta_{q}$ and $\tilde{\delta}_{q}$. The third term in Eq. (8) [13] is the gCEDM, with coefficient

$$
d_{W} \sim \mathcal{O}\left(\frac{4 \pi w}{M_{T}^{2}}\right),
$$

in terms of a dimensionless parameter $w$. The fourth and fifth operators [14, 15] are PPT FQ operators, with coefficients

$$
\operatorname{Im} \Sigma_{1,8}=\mathcal{O}\left(\frac{(4 \pi)^{2} \sigma_{1,8}}{M_{7}^{2}}\right)
$$

in terms of further dimensionless parameters $\sigma_{1,8}$. The sizes of $\delta_{0,3}, \tilde{\delta}_{0,3}, w$, and $\sigma_{1,8}$ depend on the exact mechanisms of electroweak and PT breaking and on the running to low energies where nonperturbative QCD sets in. The minimal assumption is that they are $\mathcal{O}(1), \mathcal{O}\left(g_{s} / 4 \pi\right), \mathcal{O}\left(\left(g_{s} / 4 \pi\right)^{3}\right)$, and $\mathcal{O}(1)$, respectively, with $g_{s}$ the strong coupling constant. However, they could be significantly smaller, when parameters encoding PT beyond the Standard Model are small, or significantly larger, since $f_{q}$ is unnaturally small; for discussion and examples, see for instance Refs. [2, 15].

The operators in Eqs. (7) and (8) have different transformation properties under chiral symmetry, which has profound implications for the form and relative importance of the PP pion-nucleon and $N N$ couplings in the effective Lagrangian. The $\bar{\theta}$ term in Eq. (7) transforms as the fourth component of an $S O(4)$ vector $P=\left(\bar{q} \boldsymbol{\tau} q, \bar{q} i \gamma_{5} q\right)$, the third component of which is responsible for quark-mass isospin violation [32]. PT from the $\bar{\theta}$ term and isospin violation from the quark mass difference are therefore intrinsically linked; this link appears in certain relations [10, 11, 22] between the coefficients of PPT and isospinbreaking operators in $\chi \mathrm{PT}$ through a coefficient $\rho=\left(1-\varepsilon^{2}\right) \bar{\theta} / 2 \varepsilon$. The dimension-six operators in Eq. (8) have different transformation properties still [27, 26, 23, 28, 34]. The isoscalar and isovector qEDM and qCEDM transform as the fourth and third components of two other $S O(4)$ vectors. There is no useful link to PT observables, and the third component of the qCEDM vector tends to generate hadronic interactions, which for $\bar{\theta}$ require tensor products and are of higher order. For qEDM, purely hadronic interactions arise from integrating out at least one hard photon, which leads to further breaking of chiral symmetry in the form of tensor products of the vectors with an antisymmetric chiral tensor [32. The contributions of the qEDM to hadronic couplings, like pion-nucleon or $N N$ couplings, are suppressed by $\alpha_{\mathrm{em}} / 4 \pi$. In contrast, the gCEDM and the two PPT FQ operators are singlets of the chiral group. Because they are chiral invariant, and contain no photons, the gCEDM and the two PT FQ operators lead to exactly the same effective interactions, although, of course, with different strengths. For simplicity of notation, in the following we treat gCEDM and PF FQ operators together; we refer to them as chiral-invariant $(\chi \mathrm{I})$ sources and use $w$ to denote both $w$ and $\sigma_{1,8}$ :

$$
\left\{w, \sigma_{1}, \sigma_{8}\right\} \rightarrow w
$$


We now present a subset of the complete PP chiral Lagrangian originating from the fundamental sources above. We only give the operators that play a role in the LO calculation of light-nuclei EDMs, the more general Lagrangian being found in Refs. [22, 27]. In general, a LO calculation of the EDM of a light nucleus requires six PP interactions:

$$
\begin{aligned}
\mathcal{L}_{\not P T}= & -2 \bar{N}\left(\bar{d}_{0}+\bar{d}_{1} \tau_{3}\right) S^{\mu} N v^{\nu} F_{\mu \nu}-\frac{1}{F_{\pi}} \bar{N}\left(\bar{g}_{0} \boldsymbol{\tau} \cdot \boldsymbol{\pi}+\bar{g}_{1} \pi_{3}\right) N \\
& +\bar{C}_{1} \bar{N} N \partial_{\mu}\left(\bar{N} S^{\mu} N\right)+\bar{C}_{2} \bar{N} \boldsymbol{\tau} N \cdot \partial_{\mu}\left(\bar{N} S^{\mu} \boldsymbol{\tau} N\right)+\ldots,
\end{aligned}
$$

which represent short-range isoscalar $\left(\bar{d}_{0}\right)$ and isovector $\left(\bar{d}_{1}\right)$ contributions to the nucleon EDM, isoscalar $\left(\bar{g}_{0}\right)$ and isovector $\left(\bar{g}_{1}\right)$ non-derivative pion-nucleon couplings, and two short-range PP $N N$ interactions $\left(\bar{C}_{1}, \bar{C}_{2}\right)$. Here we relegate to the "..." terms related to the above by chiral symmetry. The explicit forms of these terms depend on the PT source but, because they involve more pion fields, they do not appear in the LO EDMs we are interested in. Note that Eq. (13) is the form of $\mathcal{L}_{\not P T}$ after a field redefinition is performed to eliminate pion tadpoles and guarantee vacuum alignment; the parameters thus absorb contributions generated by this field redefinition.

Which of these six interactions is relevant depends on the system we are studying and on the fundamental PT source. As will be seen, the spin and isospin of the deuteron cause the deuteron EDM to be sensitive to only three of the above operators. In more general cases, the EDMs of light nuclei are sensitive to all six interactions. The EDMs of heavy nuclei could involve more operators than the set above. Generically one might expect a dominance by effects from $(i)$ a single nucleon, since multi-nucleon contributions tend to be suppressed at low energies by phase space; and $(i i)$ pions, thanks to their small mass and related long range. However, significant deviation from this expectation comes from the relative sizes of the various LECs, which depends on the PP source. NDA leads to the following estimates for the dimension-four and -six PPT sources:

- For the $\bar{\theta}$ term, four operators play a role at LO, the other two appearing only at subleading orders. In order to generate $\bar{g}_{1}$, which is relevant for the deuteron $\mathrm{EDM}$, the $\bar{\theta}$ term requires an insertion of the quark mass difference, which causes a suppression of $\bar{g}_{1}$ relative to $\bar{g}_{0}$ by a factor $\varepsilon m_{\pi}^{2} / M_{\mathrm{QCD}}^{2}$ [22]. (At the same order, there exists also a two-derivative pion-nucleon coupling, but for our purpose here it can be absorbed by a small change in $\bar{g}_{0}$ [34].) The LECs scale as

$$
\bar{g}_{0}=\mathcal{O}\left(\bar{\theta} \frac{m_{\pi}^{2}}{M_{\mathrm{QCD}}}\right), \quad \bar{g}_{1}=\mathcal{O}\left(\varepsilon \bar{\theta} \frac{m_{\pi}^{4}}{M_{\mathrm{QCD}}^{3}}\right), \quad \bar{d}_{0,1}=\mathcal{O}\left(e \bar{\theta} \frac{m_{\pi}^{2}}{M_{\mathrm{QCD}}^{3}}\right) .
$$

- For the qCEDM, the same four operators are needed. In this case, there is no $a$ priori relative suppression of $\bar{g}_{1}$ and the LECs scale as

$$
\begin{aligned}
\bar{g}_{0} & =\mathcal{O}\left(\left(\tilde{\delta}_{0}+\varepsilon \tilde{\delta}_{3}\right) \frac{m_{\pi}^{2} M_{\mathrm{QCD}}}{M_{T}^{2}}\right), \quad \bar{g}_{1}=\mathcal{O}\left(\tilde{\delta}_{3} \frac{m_{\pi}^{2} M_{\mathrm{QCD}}}{M_{7}^{2}}\right), \\
\bar{d}_{0,1} & =\mathcal{O}\left(\left(\tilde{\delta}_{0}+\tilde{\delta}_{3}\right) \frac{m_{\pi}^{2}}{M_{\mathrm{QCD}} M_{\tau}^{2}}\right) .
\end{aligned}
$$


(Here the "+" signs are not to be taken literally; they are only meant to signify two independent contributions to a LEC.)

- For the qEDM, only the short-range EDM contributions are important, and they scale as

$$
\bar{d}_{0,1}=\mathcal{O}\left(e \delta_{0,3} \frac{m_{\pi}^{2}}{M_{\mathrm{QCD}} M_{T}^{2}}\right) .
$$

- For the $\chi \mathrm{I}$ (gCEDM and FQ) PP sources, the non-derivative pion-nucleon interactions, which break chiral symmetry, are suppressed by a factor $m_{\pi}^{2} / M_{\mathrm{QCD}}^{2}$ compared to short-range nucleon EDM contributions and PPT $N N$ interactions, which conserve chiral symmetry. (Again, a two-derivative pion-nucleon interaction exists at the same order but can be absorbed in $\bar{g}_{0}$ [34].) All six operators thus become relevant, and the LECs scale as

$$
\begin{array}{cc}
\bar{g}_{0}=\mathcal{O}\left(w \frac{m_{\pi}^{2} M_{\mathrm{QCD}}}{M_{\mathcal{T}}^{2}}\right), & \bar{g}_{1}=\mathcal{O}\left(\varepsilon w \frac{m_{\pi}^{2} M_{\mathrm{QCD}}}{M_{\mathcal{T}}^{2}}\right), \\
\bar{d}_{0,1}=\mathcal{O}\left(e w \frac{M_{\mathrm{QCD}}}{M_{T}^{2}}\right), & \bar{C}_{1,2}=\mathcal{O}\left(w \frac{M_{\mathrm{QCD}}}{F_{\pi}^{2} M_{\mathcal{T}}^{2}}\right) .
\end{array}
$$

\subsection{EDM of the nucleon}

Using these interactions, the nucleon EDM has been calculated in $\chi \mathrm{PT}$ up to NLO for all sources of dimension up to six [25, 26, 23.

In the power counting of $\chi \mathrm{PT}$ [16], one considers typical momenta $Q \sim m_{\pi} \sim F_{\pi} \ll$ $M_{\mathrm{QCD}} \sim m_{N} \sim 2 \pi F_{\pi}$ and assigns

- a factor $Q^{4} /(4 \pi)^{2}$ for each loop integral;

- a factor $1 / Q$ for each nucleon propagator;

- a factor $1 / Q^{2}$ for each pion propagator;

- the NDA estimate for the LECs corresponding to the interactions in the diagram.

This produces for any observable an expansion in the small ratio $Q / M_{\mathrm{QCD}}$.

For the nucleon EDM, in all cases there are short-range contributions from $\bar{d}_{0,1}$ at LO. For $\mathrm{qEDM}$ and $\chi \mathrm{I}$ sources, the relative suppression of pion-nucleon couplings means that loops come at higher orders and only $\bar{d}_{0,1}$ appear up to NNLO [26]. In contrast, for $\bar{\theta}$ and qCEDM, one-loop diagrams contribute at LO and NLO. Using dimensional regularization in $d$ dimensions at a renormalization scale $\mu$, and introducing

$$
\delta \bar{d}_{1} \equiv \frac{e g_{A} \bar{g}_{0}}{\left(2 \pi F_{\pi}\right)^{2}}\left(\frac{2}{4-d}-\gamma_{E}+\ln \frac{4 \pi \mu^{2}}{m_{N}^{2}}\right)
$$


where $\gamma_{E} \simeq 0.577$ is the Euler-Mascheroni constant, the isoscalar and isovector EDMs can be expressed at NLO respectively as [25, 26, 23]

$$
d_{0}=\bar{d}_{0}+\frac{e g_{A} \bar{g}_{0}}{\left(2 \pi F_{\pi}\right)^{2}} \pi\left[\frac{3 m_{\pi}}{4 m_{N}}-\frac{\delta m_{N}}{m_{\pi}}\right]+\frac{e g_{A} \bar{g}_{1}}{\left(2 \pi F_{\pi}\right)^{2}} \frac{\pi}{4} \frac{m_{\pi}}{m_{N}}
$$

and

$$
d_{1}=\bar{d}_{1}+\delta \bar{d}_{1}+\frac{e g_{A} \bar{g}_{0}}{\left(2 \pi F_{\pi}\right)^{2}}\left[\ln \frac{m_{N}^{2}}{m_{\pi}^{2}}+\frac{5 \pi}{4} \frac{m_{\pi}}{m_{N}}-\frac{\breve{\delta} m_{\pi}^{2}}{m_{\pi}^{2}}\right]+\frac{e g_{A} \bar{g}_{1}}{\left(2 \pi F_{\pi}\right)^{2}} \frac{\pi}{4} \frac{m_{\pi}}{m_{N}},
$$

where the $\bar{g}_{1}$ terms applies to qCEDM only. The dependence on the arbitrary scale $\mu$ in $\delta \bar{d}_{1}$ is compensated by $\bar{d}_{1}$. In fact, the loop contributions cannot be separated from the short-range pieces in a model-independent way. After absorbing all these terms in $\bar{d}_{0,1}$, which we do for the rest of the paper, we can write for all sources

$$
d_{n}=\bar{d}_{0}-\bar{d}_{1}
$$

for the neutron and

$$
d_{p}=\bar{d}_{0}+\bar{d}_{1}
$$

for the proton.

However, one expects no cancellation between short-range contributions, which are analytic in $m_{\pi}^{2}$, and the "chiral-log" and other finite terms, which are not. Thus the non-analytic terms serve as lower-bound estimates for the size of $d_{p, n}$. We then expect, for $\bar{\theta}[11]$ and qCEDM [23],

$$
\begin{aligned}
& \bar{d}_{0} \gtrsim 0.01\left[\frac{\bar{g}_{0}}{F_{\pi}}+0.3 \frac{\bar{g}_{1}}{F_{\pi}}\right] \text { efm }, \\
& \bar{d}_{1} \sim 0.1\left[\frac{\bar{g}_{0}}{F_{\pi}}+0.03 \frac{\bar{g}_{1}}{F_{\pi}}\right] \text { efm } .
\end{aligned}
$$

As stressed in Ref. [26], measurements of both $d_{n}$ and $d_{p}$ alone can tell us little about the underlying source of PPT. More can be learned from measuring the EDMs of light nuclei, the calculation of which we now turn to.

\section{Ingredients: the generic case}

The EDM of a nucleus with $A \geq 2$ nucleons can be separated into two contributions. The first contribution comes from an insertion of the PPT electromagnetic current $J_{\not P T}^{0}$. The second stems from the $P T$ charge density $J_{P T}^{0}$ upon perturbing the wavefunction of the nucleus with the PPT potential $V_{P P}$, such that the wavefunction obtains a PPT component. To first order in the PPT sources, the EDM is thus a sum of two reduced matrix elements

$$
d_{A}=\frac{1}{\sqrt{6}}\left(\left\langle\Psi_{A}\left\|\vec{D}_{P P T}\right\| \Psi_{A}\right\rangle+2\left\langle\Psi_{A}\left\|\vec{D}_{P T}\right\| \widetilde{\Psi}_{A}\right\rangle\right) .
$$


The nuclear ground state $\left|\Psi_{A}\right\rangle$ and its parity admixture $\left|\widetilde{\Psi}_{A}\right\rangle$ are the solutions of homogeneous and inhomogeneous Schrödinger equations,

$$
\begin{aligned}
\left(E-H_{P T}\right)\left|\Psi_{A}\right\rangle & =0, \\
\left(E-H_{P T}\right)\left|\widetilde{\Psi}_{A}\right\rangle & =V_{P P}\left|\Psi_{A}\right\rangle,
\end{aligned}
$$

respectively, where $H_{P T}$ is the $P T$ Hamiltonian. The PPT potential $V_{\not P T}$ is shown in coordinate space in Appendix A. The EDM operators $\vec{D}_{P T}$ and $\vec{D}_{\not P T}$ are obtained from the corresponding charge densities $J_{P T}^{0}$ and $J_{\not P T}^{0}$, respectively, as discussed in Appendix $\mathrm{B}$. The factor of 2 in front of the second matrix element corresponds to the number of time-ordered diagrams, and the phases of wavefunctions are chosen so that these matrix elements are purely real.

In this section we identify the ingredients needed for the LO calculation of $d_{A}$, assuming no particular cancellations or suppressions due to spin/isospin factors.

\subsection{Power counting}

Both the potential $V_{P P T}$ and the current $J_{P P T}^{0}$ can be obtained from the Lagrangian of the previous section. The potential $V_{P P T}$ for the various PP sources has been derived in Ref. 34. To the order we are concerned with here, the potential can be taken as two-body. The PP and PT currents can also be divided into one-body and more-body currents. As we will see, the latter are dominated by two-body effects as well. There are thus four classes of contributions to a nuclear EDM, schematically drawn in Fig. 1. In order to determine which diagram(s) give(s) the most important contribution(s) we need to estimate their sizes by applying power counting.

We need to count powers of the generic momentum $Q$ in the process, in order to get an expansion in $Q / M_{\mathrm{QCD}}$. Here $Q$ is given by the nuclear binding momentum, which for a typical nucleus can be taken as $Q \sim m_{\pi} \sim F_{\pi}$, as standard in $\chi \mathrm{PT}$. However, as pointed out by Weinberg [31], the power counting of $\chi \mathrm{PT}$ needs to be adapted to the existence for $A \geq 2$ of intermediate states consisting purely of propagating nucleons. A generic diagram can be split into "reducible" parts, that contain such states, and "irreducible" subdiagrams, which do not. Within an irreducible subloop, the contour integration over the 0th component of the loop momentum can always be performed in such a way as to avoid the nucleon pole. In these diagrams the nucleon energy is of order $Q$, as assumed in $\chi \mathrm{PT}$ power counting. On the other hand, in diagrams where the intermediate state consists purely of propagating nucleons, i.e. reducible diagrams, one cannot avoid the poles of nucleon propagators, thus picking up energies $\sim Q^{2} / m_{N}$ [31] rather than $\sim Q$. Moreover, such loops also obtain an additional enhancement of $4 \pi$. The contribution of such a reducible diagram can be counted by applying the modified rules [30]:

- a factor $Q^{5} /\left(4 \pi m_{N}\right)$ for each loop integral;

- a factor $m_{N} / Q^{2}$ for each nucleon propagator;

- a factor $1 / Q^{2}$ for each pion propagator; 

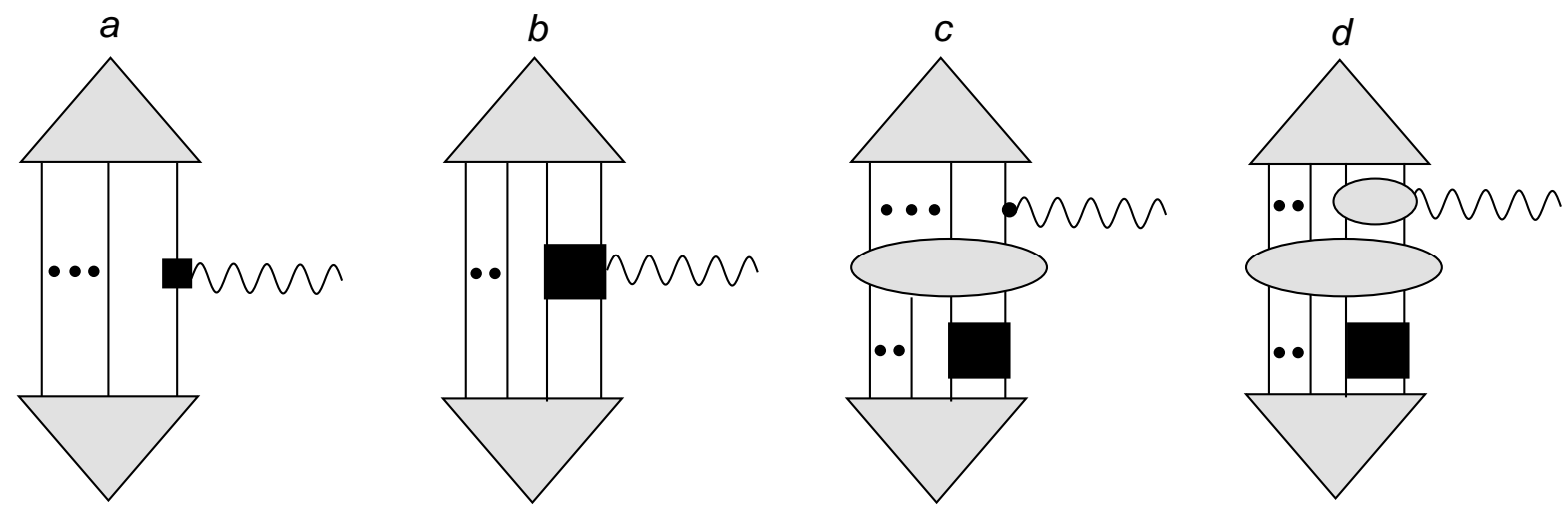

Figure 1: The four general classes of diagrams contributing to a nuclear EDM described in the text. Solid and wavy lines represent nucleons and photons. The three (two) dots stand for $A-3(A-4)$ nucleon propagators. The large triangle denotes the nuclear wavefunction; the oval, iterations of the PT potential; the dot with an attached photon, the PT one-body current; the oval with an attached photon, the PT two-body current; the black square, the PP potential; and the black square with an attached photon, the PT current.

- the NDA estimate for the LECs corresponding to the interactions in the diagram.

As an example, consider an insertion of a LO, $P T$ pion exchange in a diagram. It gives rise to one additional loop $\sim Q^{5} /\left(4 \pi m_{N}\right)$, two nucleon propagators $\sim m_{N}^{2} / Q^{4}$, a pion propagator $\sim 1 / Q^{2}$, and two insertions of the strong pion-nucleon vertex $\sim\left(Q / F_{\pi}\right)^{2}$. Combining these factors, the extra one-pion exchange amounts to $Q / M_{N N}$, where $M_{N N}=$ $4 \pi F_{\pi}^{2} / m_{N} \sim F_{\pi}$. A similar power counting holds for short-range $P T$ interactions, although the situation for them is more complicated [33]. For very light nuclei, $Q<M_{N N}$ and pion exchange can be treated perturbatively [29, 30]. The deuteron EDM has in fact already been considered in this light [26]. For less dilute nuclei, however, one expects $Q \sim M_{N N}$ and pion exchange needs to be summed to all orders [30, 33. The counting rules above are a generalization for $A \geq 2$ of the rules given in Ref. [30]. Note that they provide a natural explanation for the $Q / M_{\mathrm{QCD}}$ supression associated with an additional nucleon observed in pion-nucleus scattering [61, 35].

We can now estimate the size of each of the classes of diagrams in Fig. 11. For each class we take the PT and PT LO interactions in Eqs. (6) and (13), respectively. The iteration of the LO PT potential costs no factors, and is necessary among nucleons in reducible intermediate states, as indicated in diagrams (c) and (d) of Fig. 1. Such iteration among nucleons before and after all PT and electromagnetic insertions builds up the PT wavefunction, represented in Fig. 1 as well, which introduces an overall normalization of the diagrams. This normalization can be read off from the diagram analogous to (a), where the one-body current is given instead by the electromagnetic charge. In the following we account for this normalization by omitting the $A-1$ loops and $A+1$ nucleon propagators 
that are common to all diagrams. Thus, diagram (a) is simply

$$
D_{a}=\mathcal{O}\left(d_{p, n} Q\right)
$$

In contrast, diagram (b) has one additional irreducible loop $\sim Q^{5} /\left(4 \pi m_{N}\right)$, one additional nucleon propagator $\sim m_{N} / Q^{2}$, and the leading PT two-body current. For both qEDM and $\chi$ I sources the latter brings a suppression of a factor $Q^{2} / M_{\mathrm{QCD}}^{2}$, whereas for the other sources the contribution is comparable to the one-body term. One can continue in this fashion to find that for diagram (c),

$$
D_{c}=\mathcal{O}\left(e \frac{\bar{g}_{0,1}}{F_{\pi}^{2}} Q\right)+\mathcal{O}\left(e \bar{C}_{1,2} F_{\pi}^{2} Q\right),
$$

while for diagram (d) there is always a further suppression by a factor $Q^{2} / M_{\mathrm{QCD}}^{2}$. Analogously, more-body potentials and currents bring further suppression.

Plugging in the scaling of the LECs for the different sources, Eqs. (14), (15), (16), and (17), we can draw the following general expectations for the EDMs of light nuclei:

- For the $\bar{\theta}$ term, the nuclear EDM is dominated by diagram (c): the nuclear wavefunction acquires a PPT admixture after a one-pion exchange involving the isoscalar $\bar{g}_{0}$ vertex; the admixed wavefunction then couples to the proton charge.

- For the qCEDM, the nuclear EDM is dominated by the same effect as the $\bar{\theta}$ term. However, for the qCEDM the PPT pion-nucleon vertex can be either $\bar{g}_{0}$ or $\bar{g}_{1}$.

- For the qEDM, the nuclear EDM is dominated by the sum of the EDMs of the constituent nucleons, diagram (a).

- For $\chi \mathrm{I}$ sources, the nuclear EDM is more complicated than for the other sources. Due to the chiral suppression of the pion-nucleon interactions, diagrams (a) and (c) are equally important, and in the latter the short-range PP interactions $\bar{C}_{1,2}$ need to be included besides the one-pion exchange from both $\bar{g}_{0}$ and $\bar{g}_{1}$ couplings.

\section{$3.2 \quad P$ - and $T$-odd potential}

For all sources considered, except qEDM, an insertion of the LO PPT two-nucleon potential appears in the EDM at LO. The general PPT NN potential was derived in Ref. [34] and we summarize the relevant parts here. In momentum space the potential is given by

$$
\begin{aligned}
V_{\text {PP }}(\vec{k})= & i \frac{g_{A} \bar{g}_{0}}{F_{\pi}^{2}} \boldsymbol{\tau}^{(i)} \cdot \boldsymbol{\tau}^{(j)}\left(\vec{\sigma}^{(i)}-\vec{\sigma}^{(j)}\right) \cdot \frac{\vec{k}}{\vec{k}^{2}+m_{\pi}^{2}} \\
& +i \frac{g_{A} \bar{g}_{1}}{2 F_{\pi}^{2}}\left[\left(\tau_{3}^{(i)}+\tau_{3}^{(j)}\right)\left(\vec{\sigma}^{(i)}-\vec{\sigma}^{(j)}\right)+\left(\tau_{3}^{(i)}-\tau_{3}^{(j)}\right)\left(\vec{\sigma}^{(i)}+\vec{\sigma}^{(j)}\right)\right] \cdot \frac{\vec{k}}{\vec{k}^{2}+m_{\pi}^{2}} \\
& -\frac{i}{2}\left[\bar{C}_{1}+\bar{C}_{2} \boldsymbol{\tau}^{(i)} \cdot \boldsymbol{\tau}^{(j)}\right]\left(\vec{\sigma}^{(i)}-\vec{\sigma}^{(j)}\right) \cdot \vec{k}
\end{aligned}
$$


where $\vec{\sigma}^{(n)} / 2\left(\boldsymbol{\tau}^{(n)} / 2\right)$ is the spin (isospin) vector of nucleon $n$, and $\vec{k}=\vec{p}_{i}-\vec{p}_{i}^{\prime}$ is the momentum transferred from nucleon $i$. In this expression, at LO $\bar{g}_{0}$ originates from $\bar{\theta}-$ term, qCEDM, and $\chi \mathrm{I}$ sources; $\bar{g}_{1}$ from qCEDM and $\chi \mathrm{I}$ sources; and $\bar{C}_{i}$ from $\chi \mathrm{I}$ sources only. The pion-exchange parts are well known (for example, Refs. [50, 38, 39]), while the contact interactions incorporate all other PT effects of short-range, such as single exchanges of the mesons $\omega$ and $\eta\left(\bar{C}_{1}\right)$ and $\rho\left(\bar{C}_{2}\right)$ [34].

\subsection{Currents}

As we argued above, only one-body currents are necessary at LO. For the $\bar{\theta}$ term, qCEDM, and $\chi \mathrm{I}$ sources we need the PT current coming from the proton charge in Eq. (6),

$$
J_{P T}^{0}=\frac{e}{2}\left(1+\tau_{3}^{(i)}\right)
$$

where $\boldsymbol{\tau}^{(i)} / 2$ is the isospin of the nucleon that couples to the one-body current.

For the qEDM and $\chi \mathrm{I}$ sources we need as well the PPT current originating from the nucleon EDMs,

$$
J_{\not P T}^{0}=-i\left(\bar{d}_{0}+\bar{d}_{1} \tau_{3}^{(i)}\right) \vec{\sigma}^{(i)} \cdot \vec{q}
$$

where $\vec{\sigma}^{(i)} / 2$ is the spin of the nucleon that interacts with the photon and $\vec{q}$ is the outgoing photon momentum.

\section{Ingredients: nuclei with $N=Z$}

Although the power counting discussed above holds for general light nuclei, it is possible that a diagram, which is expected to be LO, does not contribute to the EDMs of certain systems. For nuclei of equal neutron and proton number, $N=Z$, i.e. the third component of isospin $I_{3}=0$, an insertion of the isoscalar PPT potential in combination with the LO one-body PT current, i.e. Eq. (31), does not contribute to the EDM [50]. To see this, consider the EDM operator resulting from the LO one-body PT current, which takes the simple expression

$$
\vec{D}_{P T}^{(1)}=\frac{e}{2} \sum_{i=1}^{A}\left(1+\tau_{3}^{(i)}\right) \vec{\xi}_{i}=\frac{e}{2} \sum_{i=1}^{A} \tau_{3}^{(i)} \vec{\xi}_{i}
$$

in intrinsic coordinates $\vec{\xi}_{i}$ with $\sum_{i=1}^{A} \vec{\xi}_{i}=0$. Since this operator is isovector, i.e. $\Delta I=$ 1 , and conserves $I_{3}$, i.e. $\Delta I_{3}=0$, it can only yield a non-vanishing moment when the nuclear state of a $\left(I, I_{3}=0\right)$ nucleus acquires some parity admixture with isospin $\left(I^{\prime}=I \pm 1, I_{3}^{\prime}=0\right)$. Therefore, one needs isovector components in $V_{P P T}$ to induce such admixture. The above argument holds in the non-relativistic limit.

This observation is of no concern for sources where there are other contributions at the same order as those contributions that vanish. The nuclear EDM is then simply 
dominated by the non-vanishing LO terms. For the $\bar{\theta}$ term, however, the LO contribution consists only of an insertion of the isoscalar PPT potential, such that, for $N=Z$ nuclei, we need to go further down in power counting to find the dominant EDM contributions.

\subsection{Power counting}

Because the formally leading diagram (c) of Fig. 1 vanishes for $N=Z$ in the $\bar{\theta}$-term case when both the PT one-body current and the PT two-body potential are used, let us first consider corrections in this diagram. It turns out that NLO corrections to both the PP potential [34] and PT one-body current vanish, and the first corrections we need to account for are at NNLO. By looking at the scaling of the LECs for the $\bar{\theta}$ term in Eq. (14) and the power counting for the classes of diagrams in Fig. 1, we then conclude that the first non-vanishing contributions can come from all classes of diagrams: the LO nucleon EDMs in diagram (a), the LO PPT two-body currents in diagram (b), the NNLO PT two-body potential or the NNLO PT one-body current in diagram (c), and the LO $P T$ two-body currents with the LO PPT two-body potential in diagram (d).

For the other sources only parts of the LO contributions given in the previous section remain. For qCEDM and $\chi \mathrm{I}$ sources we need the PPT potential from $\bar{g}_{1}$ OPE. For qEDM and $\chi \mathrm{I}$ sources we also need the isoscalar short-range contribution to the nucleon EDM.

\section{2 $\quad P$ - and $T$-odd potential}

For $\mathrm{qCEDM}$ and $\chi \mathrm{I}$ sources we can use the same potential as in the generic case, but the $\bar{g}_{0}$ and $\bar{C}_{1,2}$ terms will not contribute. We do not require a PT-potential for qEDM. For the $\bar{\theta}$ term we need the NNLO PPT potential calculated in Ref. [34]. At this order further isoscalar terms appear, which also will not contribute. Thus we need here only the following terms:

$$
\begin{aligned}
V_{\text {PPT }}(\vec{k}, \vec{K}, \vec{P})= & \frac{i}{2 F_{\pi}^{2}}\left[\left(g_{A} \bar{g}_{1}-\frac{\bar{g}_{0} \beta_{1}}{2}\right)\left(\tau_{3}^{(i)}+\tau_{3}^{(j)}\right)\left(\vec{\sigma}^{(i)}-\vec{\sigma}^{(j)}\right)\right. \\
& \left.+\left(g_{A} \bar{g}_{1}+\frac{\bar{g}_{0} \beta_{1}}{2}\right)\left(\tau_{3}^{(i)}-\tau_{3}^{(j)}\right)\left(\vec{\sigma}^{(i)}+\vec{\sigma}^{(j)}\right)\right] \cdot \frac{\vec{k}}{\vec{k}^{2}+m_{\pi}^{2}} \\
& +i \frac{\bar{g}_{0} g_{A}}{3 F_{\pi}^{2}}\left[\breve{\delta} m_{\pi}^{2}-\delta m_{\pi}^{2}-\frac{\left(\breve{\delta} m_{\pi}^{2}\right)^{2}}{\vec{k}^{2}+m_{\pi}^{2}}-\delta m_{N}^{2}\right]\left(3 \tau_{3}^{(i)} \tau_{3}^{(j)}-\boldsymbol{\tau}^{(i)} \cdot \boldsymbol{\tau}^{(j)}\right) \\
& \times\left(\vec{\sigma}^{(i)}-\vec{\sigma}^{(j)}\right) \cdot \frac{\vec{k}}{\left(\vec{k}^{2}+m_{\pi}^{2}\right)^{2}}+\frac{\bar{g}_{0} g_{A}}{F_{\pi}^{2}} \frac{\delta m_{N}}{m_{N}}\left(\boldsymbol{\tau}^{(i)} \times \boldsymbol{\tau}^{(j)}\right)_{3} \\
& \times\left[\left(\vec{\sigma}^{(i)}+\vec{\sigma}^{(j)}\right) \cdot \vec{K}+\left(\vec{\sigma}^{(i)}-\vec{\sigma}^{(j)}\right) \cdot\left(\frac{\vec{P}}{2}+\frac{(\vec{P} \cdot \vec{k}) \vec{k}}{\vec{k}^{2}+m_{\pi}^{2}}\right)\right] \frac{1}{\vec{k}^{2}+m_{\pi}^{2}}(34)
\end{aligned}
$$

where $\vec{P}=\vec{p}_{i}+\vec{p}_{j}$ is the center-of-mass (CM) momentum of the nucleon pair and $\vec{K}=$ $\left(\vec{p}_{i}+\vec{p}_{i}^{\prime}-\vec{p}_{j}-\vec{p}_{j}^{\prime}\right) / 4$. The first two terms originate in one-pion exchange with $\bar{g}_{1}$ instead 
of $\bar{g}_{0}$ or with $\beta_{1}$ instead of $g_{A}$. The next term arises from isospin breaking in the pion and nucleon masses, and it is very small [34]. The last term is due to isospin breaking in the pion-nucleon vertex. The potential also includes $1 / m_{N}^{2}$ corrections [34], which we do not include here for the reasons given below.

\subsection{Currents}

For the same reasons that require the NNLO PPT potential we also need the NNLO PT onebody electric current, to be used with the $\bar{\theta}$-term LO potential. Again we do not bother with terms that give a vanishing contribution for $N=Z$ nuclei. The only remaining correction from Eq. (6) is given by

$$
J_{P T}^{0}=-\frac{i e}{16 m_{N}^{2}} \varepsilon^{l m n} \sigma^{(i) l} q^{m}\left(p_{i}+p_{i}^{\prime}\right)^{n}\left[1+2 \kappa_{0}+\left(1+2 \kappa_{1}\right) \tau_{3}^{(i)}\right]
$$

which agrees with Ref. [60]. Here $\vec{p}_{i}\left(\vec{p}_{i}^{\prime}\right)$ is the momentum of the nucleon that couples to the photon before (after) interaction.

We also need two-body currents, both PT and PPT. We use incoming momenta $\vec{p}_{i}=$ $\vec{P} / 2+\vec{p}$ and $\vec{p}_{j}=\vec{P} / 2-\vec{p}$ and outgoing momenta $\vec{p}_{i}^{\prime}=\vec{P}^{\prime} / 2+\vec{p}^{\prime}$ and $\vec{p}_{j}^{\prime}=\vec{P}^{\prime} / 2-\vec{p}^{\prime}$. The photon momentum $\vec{q}=\vec{P}-\vec{P}^{\prime}$ is outgoing. For convenience we introduce $\vec{k}=\vec{p}-\vec{p}^{\prime}$ as before, $\vec{K}=\left(\vec{p}+\vec{p}^{\prime}\right) / 2$, and $\vec{P}_{t}=\left(\vec{P}+\vec{P}^{\prime}\right) / 2$. In the evaluation of the currents at the order we are interested we can use the nucleon on-shell relation $p_{n}^{0}=\vec{p}_{n}^{2} / 2 m_{N}$, or alternatively $k^{0}=\left(\overrightarrow{P_{t}} \cdot \vec{k}-\vec{q} \cdot \vec{K}\right) / 2 m_{N}$.

The relevant diagrams for the LO two-body $P T$ electric current, used again in combination with the LO PPT two-body potential, are shown in Fig. 2. All interactions come from the PT Lagrangian, Eq. (6). In momentum space the current reads

$$
\begin{aligned}
J_{P T, a}^{0}= & +\frac{2 i e g_{A}^{2}}{F_{\pi}^{2}}\left(\boldsymbol{\tau}^{(i)} \times \boldsymbol{\tau}^{(j)}\right)_{3} k^{0} \frac{\left[\vec{\sigma}^{(i)} \cdot(\vec{k}+\vec{q} / 2)\right]\left[\vec{\sigma}^{(j)} \cdot(\vec{k}-\vec{q} / 2)\right]}{\left[(\vec{k}+\vec{q} / 2)^{2}+m_{\pi}^{2}\right]\left[(\vec{k}-\vec{q} / 2)^{2}+m_{\pi}^{2}\right]}, \\
J_{P T, b}^{0}= & -\frac{i e g_{A}^{2}}{2 F_{\pi}^{2} m_{N}}\left(\boldsymbol{\tau}^{(i)} \times \boldsymbol{\tau}^{(j)}\right)_{3} \\
& \times\left[\frac{\left[\vec{\sigma}^{(i)} \cdot\left(\vec{P}_{t}+2 \vec{K}\right)\right]\left[\vec{\sigma}^{(j)} \cdot(\vec{k}-\vec{q} / 2)\right]}{(\vec{k}-\vec{q} / 2)^{2}+m_{\pi}^{2}}+\frac{\left[\vec{\sigma}^{(j)} \cdot\left(\overrightarrow{P_{t}}-2 \vec{K}\right)\right]\left[\vec{\sigma}^{(i)} \cdot(\vec{k}+\vec{q} / 2)\right]}{(\vec{k}+\vec{q} / 2)^{2}+m_{\pi}^{2}}\right], \\
J_{P T, c}^{0}= & -\frac{2 e g_{A}^{2}}{F_{\pi}^{2}} \delta m_{N}\left(\boldsymbol{\tau}^{(i)} \cdot \boldsymbol{\tau}^{(j)}-\tau_{3}^{(i)} \tau_{3}^{(j)}\right) \frac{\left[\vec{\sigma}^{(i)} \cdot(\vec{k}+\vec{q} / 2)\right]\left[\vec{\sigma}^{(j)} \cdot(\vec{k}-\vec{q} / 2)\right]}{\left[(\vec{k}+\vec{q} / 2)^{2}+m_{\pi}^{2}\right]\left[(\vec{k}-\vec{q} / 2)^{2}+m_{\pi}^{2}\right]} .
\end{aligned}
$$

We also need to include the LO two-nucleon PPT electric current. The diagrams contributing to this current are shown in Fig. 3. Here $P T$ interactions come from the $P T$ Lagrangian, Eq. (6), and the PP interaction is the $\bar{g}_{0}$ vertex in the PPT Lagrangian, Eq. 

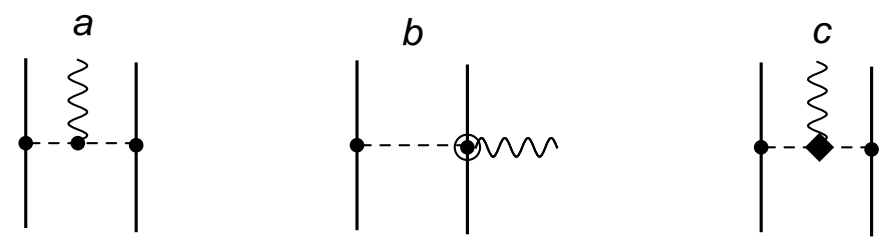

Figure 2: Diagrams contributing to the PT two-nucleon electric current. Solid, dashed, and wavy lines represent nucleons, pions, and photons. A diamond marks an isospinbreaking $P T$ interaction and the other vertices isospin-conserving $P T$ interactions: leading (filled circles) and subleading (circled circles). Only one topology per diagram is shown.
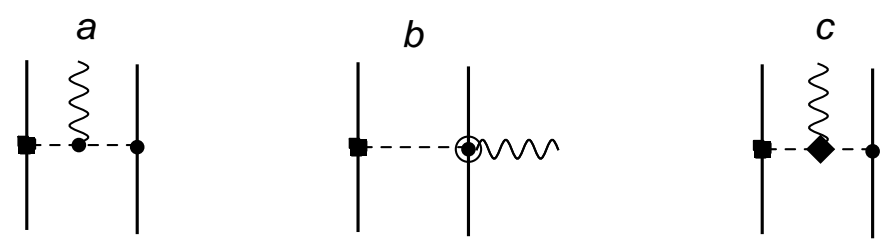

Figure 3: Diagrams contributing to the PT two-nucleon electric current. A square marks a PP interaction; other notation as in Fig. 2. Only one topology per diagram is shown.

(13). The current is given by

$$
\begin{aligned}
& J_{\text {PP }, a}^{0}=+\frac{2 e g_{A} \bar{g}_{0}}{F_{\pi}^{2}}\left(\boldsymbol{\tau}^{(i)} \times \boldsymbol{\tau}^{(j)}\right)_{3} k^{0} \frac{\left(\vec{\sigma}^{(i)}+\vec{\sigma}^{(j)}\right) \cdot \vec{q} / 2+\left(\vec{\sigma}^{(i)}-\vec{\sigma}^{(j)}\right) \cdot \vec{k}}{\left[(\vec{k}+\vec{q} / 2)^{2}+m_{\pi}^{2}\right]\left[(\vec{k}-\vec{q} / 2)^{2}+m_{\pi}^{2}\right]}, \\
& J_{\text {PP }, b}^{0}=-\frac{e g_{A} \bar{g}_{0}}{2 F_{\pi}^{2} m_{N}}\left(\boldsymbol{\tau}^{(i)} \times \boldsymbol{\tau}^{(j)}\right)_{3}\left[\frac{\vec{\sigma}^{(i)} \cdot\left(\vec{P}_{t}+2 \vec{K}\right)}{(\vec{k}-\vec{q} / 2)^{2}+m_{\pi}^{2}}-\frac{\vec{\sigma}^{(j)} \cdot\left(\vec{P}_{t}-2 \vec{K}\right)}{(\vec{k}+\vec{q} / 2)^{2}+m_{\pi}^{2}}\right], \\
& J_{P P T, c}^{0}=+\frac{2 i e g_{A} \bar{g}_{0}}{F_{\pi}^{2}} \delta m_{N}\left(\boldsymbol{\tau}^{(i)} \cdot \boldsymbol{\tau}^{(j)}-\tau_{3}^{(i)} \tau_{3}^{(j)}\right) \frac{\left(\vec{\sigma}^{(i)}+\vec{\sigma}^{(j)}\right) \cdot \vec{q} / 2+\left(\vec{\sigma}^{(i)}-\vec{\sigma}^{(j)}\right) \cdot \vec{k}}{\left[(\vec{k}+\vec{q} / 2)^{2}+m_{\pi}^{2}\right]\left[(\vec{k}-\vec{q} / 2)^{2}+m_{\pi}^{2}\right]} .
\end{aligned}
$$

\section{$5 \quad$ EDM of the Deuteron}

We are now in position to calculate the EDM of the deuteron, which provides the simplest example of an $N=Z$ nucleus. The ground state of the deuteron is mainly a ${ }^{3} S_{1}$ state. The deuteron obtains a ${ }^{1} P_{1}$ component after a $\bar{g}_{0}$ pion exchange or an insertion of $\bar{C}_{1,2}$. Since the LO PT one-nucleon current is spin independent, it cannot bring the deuteron wavefunction from ${ }^{1} P_{1}$ to ${ }^{3} S_{1}$, and therefore these contributions vanish for the deuteron, as anticipated on more general grounds in the previous section.

The deuteron EDM has been studied before in the meson-exchange picture [36, 37, 38, 39, 40, with various degrees of sophistication in the treatments of the $P$ - and $T$ conserving interaction $H_{P T}$. Using modern high-quality phenomenological potentials [44, 45], Ref. [39] found that the model dependence of $H_{P T}$ is rather small for a deuteron 
EDM generated by the OPE sector of the PPT interaction. The detailed study in Ref. [40] confirmed this point. Since our new EFT scheme shows that the leading-order contribution from various PPT sources to the deuteron EDM also comes from the long-range terms in $V_{P P T}$, we take advantage of the existing calculation scheme of Ref. [39] to obtain wave functions $\left|\Psi_{2 \mathrm{H}}\right\rangle$ and $\left|\widetilde{\Psi}_{{ }_{2} \mathrm{H}}\right\rangle$. The calculation is performed in coordinate space using the PP potentials and currents from Appendices $\mathrm{A}$ and B, respectively. Of course, a fully consistent treatment would involve using the $P T$ interaction $H_{P T}$ derived from the complete chiral Lagrangian, instead of a phenomenological potential. At present, unfortunately, such a consistent potential does not exist beyond LO [33]. It would include relativistic corrections as well, which are absent in the phenomenological potentials we use. For this reason, we neglect relativistic corrections in the PT potential and currents as well. We expect that the results from a fully consistent calculation will not deviate significantly from the results we obtain here. The numbers below correspond to the Argonne $v_{18}$ potential [45], but results for the Reid93 and Nijmegen II potentials [44] agree within 5\%. This is less than the error of order $m_{\pi} / M_{\mathrm{QCD}} \sim 20 \%$ intrinsic to $\chi \mathrm{PT}$ in lowest order.

The simplest contribution to the deuteron EDM comes from the constituent EDMs. The LO $J_{P P T}^{0}$, given in Eq. 32 , yields a one-body EDM operator

$$
\vec{D}_{\not P T}^{(1)}=\sum_{i=1}^{A}\left(\bar{d}_{0}+\bar{d}_{1} \tau_{3}^{(i)}\right) \vec{\sigma}^{(i)} .
$$

For the deuteron, an isoscalar $(I=0)$ and spin-triplet $(S=1)$ state, one simply gets

$$
\frac{1}{\sqrt{6}}\left\langle\Psi_{2_{\mathrm{H}}}\left\|\vec{D}_{\not P T}^{(1)}\right\| \Psi_{{ }^{\mathrm{H}}}\right\rangle=d_{n}+d_{p} .
$$

In order for $\vec{D}_{P T}^{(1)}$, a purely isovector operator as discussed earlier, to yield a non-zero contribution in the deuteron, it is obvious that the parity admixture $\left|\widetilde{\Psi}_{2_{\mathrm{H}}}\right\rangle$ has to be a ${ }^{3} P_{1}$ state. Among the various terms in the LO PPT potential, Eq. (30), only the one with the isospin-spin operator $\left(\tau_{3}^{(1)}-\tau_{3}^{(2)}\right)\left(\vec{\sigma}^{(1)}+\vec{\sigma}^{(2)}\right)$ can contribute. The result is

$$
\frac{2}{\sqrt{6}}\left\langle\Psi_{2 \mathrm{H}}\left\|\vec{D}_{P T}^{(1)}\right\| \widetilde{\Psi}_{{ }^{2} \mathrm{H}}\left({ }^{3} P_{1}\right)\right\rangle=-0.19 \frac{\bar{g}_{1}}{F_{\pi}} \text { e fm } .
$$

However, when it comes to the $\bar{\theta}$ term, because the LO contribution vanishes as argued in the previous section, the leading contribution is in fact NNLO. Among the higher-order interactions identified in Section 4.2 , the terms with coupling constants $\left(g_{A} \bar{g}_{1}+\bar{g}_{0} \beta_{1} / 2\right)$ and $g_{A} \bar{g}_{0} \delta m_{N}$ in Eq. (34) can contribute, by isospin and spin selection rules. Except for the coupling constants, the operator structures of the former are the same as the one in Eq. (30), so the matrix element can simply be obtained by replacing

$$
\bar{g}_{1} \rightarrow \bar{g}_{1}+\frac{\beta_{1}}{2 g_{A}} \bar{g}_{0}
$$


in Eq. (40). Combining this with the contribution from the isospin-breaking pion-nucleon vertex, we find the matrix element

$$
\frac{2}{\sqrt{6}}\left\langle\Psi_{2 \mathrm{H}}\left\|\vec{D}_{P T}^{(1)}\right\| \widetilde{\Psi}_{2 \mathrm{H}}\left({ }^{3} P_{1}\right)\right\rangle=-\left[0.19\left(\frac{\bar{g}_{1}}{F_{\pi}}+\frac{\beta_{1}}{2 g_{A}} \frac{\bar{g}_{0}}{F_{\pi}}\right)+5.8 \cdot 10^{-4} \frac{\bar{g}_{0}}{F_{\pi}}\right] \text { efm }
$$

for the $\bar{\theta}$ term.

For the $\bar{\theta}$ term, there are in addition NNLO currents to be taken into account. For the PF currents, as the corresponding EDM operators are sandwiched between two isoscalar states, they must be isoscalar to contribute. Among the NNLO PT currents identified in Section 4.3, only the third current in Eq. (38), $J_{\not P T, c}^{0}$, meets the requirement and leads to a two-body EDM operator (see Appendix B)

$$
\vec{D}_{\text {PPT }}^{(2)}=-e \frac{g_{A} \bar{g}_{0}}{F_{\pi}^{2}} \delta m_{N}\left(\boldsymbol{\tau}^{(1)} \cdot \boldsymbol{\tau}^{(2)}-\tau_{3}^{(1)} \tau_{3}^{(2)}\right)\left[\vec{\sigma}^{(1)} \cdot \vec{\nabla}_{1}+\vec{\sigma}^{(2)} \cdot \vec{\nabla}_{2},\left(\vec{x}_{1}+\vec{x}_{2}\right) \frac{e^{-m_{\pi}\left|\vec{x}_{1}-\vec{x}_{2}\right|}}{8 \pi m_{\pi}}\right]
$$

in terms of the positions $\vec{x}_{1}$ and $\vec{x}_{2}$ of the two nucleons and the derivatives $\vec{\nabla}_{1}$ and $\vec{\nabla}_{2}$ with respect to them. This results in the matrix element

$$
\frac{1}{\sqrt{6}}\left\langle\Psi_{2 \mathrm{H}}\left\|\vec{D}_{\not P T}^{(2)}\right\| \Psi_{2 \mathrm{H}}\right\rangle=1.1 \cdot 10^{-3} \frac{\bar{g}_{0}}{F_{\pi}} \text { efm }
$$

for the deuteron EDM. The contributions of two-body PT currents to the EDM have again to be coupled with the parity admixture generated by the LO $V_{P P}$, which is purely isoscalar when $\bar{\theta}$ is the PPT source. The only PT current with an isoscalar component, among those identified in Section 4.3, is the third current in Eq. (36), $J_{P T, c^{*}}^{0}$ It gives a two-body EDM operator

$\vec{D}_{P T}^{(2)}=-e \frac{g_{A}^{2}}{F_{\pi}^{2}} \delta m_{N}\left(\boldsymbol{\tau}^{(1)} \cdot \boldsymbol{\tau}^{(2)}-\tau_{3}^{(1)} \tau_{3}^{(2)}\right)\left[\left(\vec{\sigma}^{(1)} \cdot \vec{\nabla}_{1}\right)\left(\vec{\sigma}^{(2)} \cdot \vec{\nabla}_{2}\right),\left(\vec{x}_{1}+\vec{x}_{2}\right) \frac{e^{-m_{\pi}\left|\vec{x}_{1}-\vec{x}_{2}\right|}}{8 \pi m_{\pi}}\right]$.

Since the isoscalar parity admixture $\left|\widetilde{\Psi}_{2 \mathrm{H}}\right\rangle$ can only be a ${ }^{1} P_{1}$ state, this current gives a matrix element

$$
\frac{2}{\sqrt{6}}\left\langle\Psi_{2 \mathrm{H}}|| \vec{D}_{P T}^{(2)} \| \widetilde{\Psi}_{{ }^{2} \mathrm{H}}\left({ }^{1} P_{1}\right)\right\rangle=-3.3 \cdot 10^{-4} \frac{\bar{g}_{0}}{F_{\pi}} \text { efm } .
$$

In total the deuteron EDM can be written as a function of three PPT LECs,

$$
d_{2_{\mathrm{H}}}=d_{p}+d_{n}+\left[-0.19 \frac{\bar{g}_{1}}{F_{\pi}}+\left(0.2-0.7 \cdot 10^{2} \beta_{1}\right) \cdot 10^{-3} \frac{\bar{g}_{0}}{F_{\pi}}\right] \text { efm }
$$

where $d_{p, n}$ should be included for $\bar{\theta}$, qEDM, and $\chi \mathrm{I} ; \bar{g}_{1}$ for $\bar{\theta}$, qCEDM, and $\chi \mathrm{I}$; and $\bar{g}_{0}$ for $\bar{\theta}$ only.

This result can be compared, for each of the sources, with the calculation where OPE is treated perturbatively [28]. For both qCEDM and qEDM the nonperturbative pion 
approach adopted here agrees very well with the perturbative calculation. In the case of the qCEDM, it was also found that the deuteron EDM is dominated by $\bar{g}_{1}$ pion exchange and given by [28]

$$
\left.d_{2_{\mathrm{H}}}(\mathrm{qCEDM})\right|_{\text {pert }}=-\frac{e g_{A} \bar{g}_{1} m_{N}}{6 \pi F_{\pi}^{2} m_{\pi}} \frac{1+\gamma / m_{\pi}}{\left(1+2 \gamma / m_{\pi}\right)^{2}}=-0.23 \frac{\bar{g}_{1}}{F_{\pi}} \text { efm }
$$

where $\gamma \simeq 45 \mathrm{MeV}$ is the binding momentum of the deuteron. This result agrees exactly with a zero-range model [38] and is $22 \%$ larger than the result from the qCEDM calculation with nonperturbative OPE [39] reproduced above,

$$
d_{2 \mathrm{H}}(\mathrm{qCEDM})=-0.19 \frac{\bar{g}_{1}}{F_{\pi}} \text { efm } .
$$

Since the estimated error in the perturbative calculation is of order $Q / M_{N N} \sim 30 \%$, the calculations agree within their uncertainty. By power counting the contribution from $\bar{d}_{0}$ is expected to be suppressed by $m_{\pi}^{2} / M_{\mathrm{QCD}}^{2}$ compared to Eq. 49). From Eq. (15) we infer $\bar{d}_{0} F_{\pi} / \bar{g}_{1}=\mathcal{O}\left(e F_{\pi} / M_{\mathrm{QCD}}^{2}\right) \sim 0.03 e \mathrm{fm}$, implying that, in the case of qCEDM, the nucleon EDMs contribute at the $30 \%$ level to the deuteron EDM. This suppression is less than formally expected. If we assume the isoscalar nucleon EDM is saturated by its long-range part, Eq. 23), the contribution is at the $10 \%$ level. In any case, the correction by the isoscalar nucleon EDM is of the order of the intrinsic $\chi \mathrm{PT}$ uncertainty $m_{\pi} / M_{\mathrm{QCD}}$, such that for the qCEDM the deuteron EDM at LO is given by Eq. (49).

Likewise, for qEDM the conclusions of Ref. [28] do not change once we treat OPE nonperturbatively. The deuteron EDM is in this case simply the sum of the neutron and proton EDM,

$$
d_{2 \mathrm{H}}(\mathrm{qEDM})=2 \bar{d}_{0} .
$$

The comparison is more subtle for $\bar{\theta}$ and $\chi \mathrm{I}$ PP sources. For both of these sources, the deuteron EDM is expected in the perturbative-pion approach to be dominated by the isoscalar nucleon EDM, since pion exchange is further suppressed in the $Q / M_{N N}$ expansion. In the nonperturbative power counting PT pion exchange is a dominant effect as well. In order to compare the two effects - nucleon EDMs and pion exchange - in the nonperturbative calculation we can look at the estimated scaling of the LECs. For $\chi \mathrm{I}$ sources,

$$
d_{2 \mathrm{H}}(\chi \mathrm{I})=2 \bar{d}_{0}-0.19 \frac{\bar{g}_{1}}{F_{\pi}} \text { efm } .
$$

From Eq. (17) we infer that $F_{\pi} \bar{d}_{0} / \bar{g}_{1}=\mathcal{O}\left(e F_{\pi} / \varepsilon m_{\pi}^{2}\right) \sim 5 e$ fm. Thus, although formally $\bar{g}_{1}$ exchange is LO, because of a combination of $\varepsilon$ suppression and the relatively small factor of 0.19 in Eq. (47), it actually is expected to contribute only at the $\sim 5 \%$ level to the deuteron EDM. For $\bar{\theta}$ there are additional contributions from $\bar{g}_{0}$,

$$
d_{2 \mathrm{H}}(\bar{\theta})=2 \bar{d}_{0}+\left[-0.19 \frac{\bar{g}_{1}}{F_{\pi}}+\left(0.2-0.7 \cdot 10^{2} \beta_{1}\right) \cdot 10^{-3} \frac{\bar{g}_{0}}{F_{\pi}}\right] \text { efm . }
$$

The contributions from the PT and PT two-body currents, Eqs. (43) and 45) respectively, are of similar size. The $N N$ data constraint [59] on $\beta_{1}$ shows that the contribution from 
the PP potential is no larger, and the full $\bar{g}_{0}$ term is $\lesssim 0.9 \cdot 10^{-3}\left(\bar{g}_{0} / F_{\pi}\right)$ efm. From Eq. (14) we expect that $\bar{g}_{1} / \bar{g}_{0}=\mathcal{O}\left(\varepsilon m_{\pi}^{2} / M_{\mathrm{QCD}}^{2}\right) \sim 10^{-2}$, so the $\bar{g}_{1} / F_{\pi}$ contribution should be comparable to these small $\bar{g}_{0} / F_{\pi}$ contributions. In contrast, we expect a larger weight from the pion cloud around each nucleon, which for $\bar{d}_{0}$ enters at NLO and gives Eq. 23. Thus again, although pion-exchange contributions in the potential and currents are formally LO, $\varepsilon$ suppression and relatively small numerical factors in the deuteron make them likely no more than $\sim 10 \%$ of the nucleon EDM contribution.

The fact that pion-exchange contributions are expected to be smaller in the deuteron than assumed in $\chi \mathrm{PT}$ power counting confirms that the power counting of Ref. [28], where pion exchange comes in at NLO, works better for a loosely bound nucleus. The $\chi \mathrm{PT}$ power counting should become more accurate as we consider heavier, denser nuclei, the simplest of which we tackle next.

\section{EDM of the Helion and the Triton}

In this section we investigate the EDMs of ${ }^{3} \mathrm{He}$ and ${ }^{3} \mathrm{H}$. No particular cancellations are expected, so the framework of Section 3 applies.

The EDM of ${ }^{3}$ He was studied in Ref. [42], where two PPT mechanisms were considered: nucleon EDMs and a PT two-nucleon potential containing the most general non-derivative, single $\pi-, \rho$, and $\omega$-meson exchanges. The nuclear wavefunction was calculated with the no-core shell model (NCSM) 62], where a PT nuclear potential is solved within a model space made from appropriately symmetrized combinations [63] of $N_{\max }$ harmonicoscillator wavefunctions of frequency $\Omega$. In Ref. [42] both Argonne $v 18$ [45] and EFTinspired [46] potentials, including the Coulomb interaction, were used. At large enough $N_{\max }$ results become independent of $\Omega$.

Here we adapt this calculation to the PF ingredients from chiral EFT, and calculate the EDM of ${ }^{3} \mathrm{H}$ for the first time. As argued in Section 3, power counting for generic light nuclei tells us that for all PPT sources of dimension up to six, the EDM is indeed expected to come mostly from the nucleon EDM and from the two-nucleon PP potential, as assumed in Ref. [42]. The only difference is that the EFT potential (30) contains, in addition to OPE, also two LECs $\left(\bar{C}_{1}\right.$ and $\left.\bar{C}_{2}\right)$ representing shorter-range interactions. This potential in coordinate space is given in Appendix A. The OPE terms were included in Ref. [42, while $\bar{C}_{1}$ and $\bar{C}_{2}$ can be thought of as originating from, respectively, $\omega$ and $\rho$ exchanges, also considered there. The relation can be made quite explicit if we choose to regularize the delta functions with Yukawa functions, following a strategy successfully employed before to study the effects of the EFT PPT potential [49]:

$$
\begin{aligned}
\frac{m_{1}^{2} \bar{C}_{1}}{4 \pi r} e^{-m_{1} r} & \rightarrow \bar{C}_{1} \delta^{(3)}(\vec{r}), \\
\frac{m_{2}^{2} \bar{C}_{2}}{4 \pi r} e^{-m_{2} r} & \rightarrow \bar{C}_{2} \delta^{(3)}(\vec{r}),
\end{aligned}
$$

as $m_{1,2} \rightarrow \infty$. When $m_{1}=m_{\omega}\left(m_{2}=m_{\rho}\right)$ and $\bar{C}_{1}\left(\bar{C}_{2}\right)$ is an appropriate combination of $\omega(\rho)$ couplings [34], the expressions on the left-hand side coincide with those in Ref. [42]. 

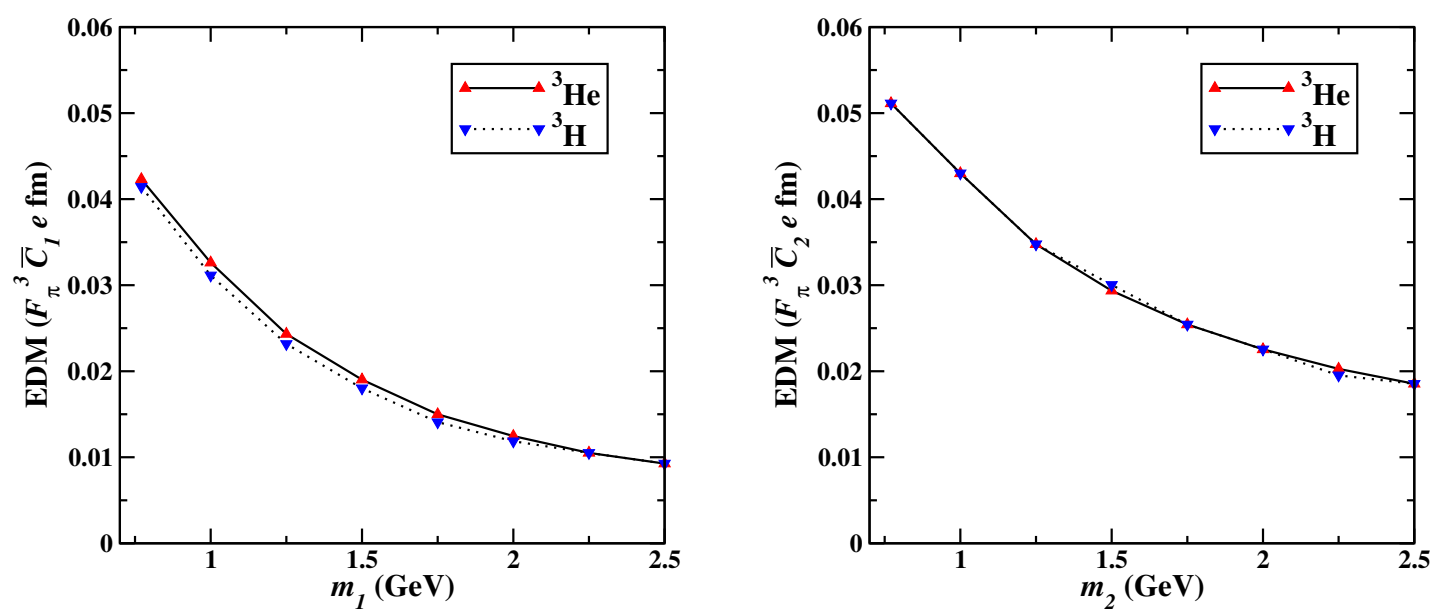

Figure 4: Magnitude of the tri-nucleon EDMs in units of $F_{\pi}^{3} \bar{C}_{i} e \mathrm{fm}$, as function of the regulator mass in GeV: $i=1$ (left panel) and $i=2$ (right panel). The solid (dashed) curve is for helion (triton).

Here we recalculate these contributions for values of $m_{1,2}$ up to $2.5 \mathrm{GeV}$. For uniformity with Section 5 we again display numbers obtained with the Argonne $v 18$ potential. In Ref. [42] it was found that for helion the contributions from nucleon EDMs $\left(\bar{d}_{0,1}\right)$ and from pion exchange $\left(\bar{g}_{0,1}\right)$ change with $P T$ potential by no more than $\sim 25 \%$. We have verified that the same is true for triton. Unfortunately the situation is different for the short-range two-body contributions $\left(\bar{C}_{1,2}\right)$, which are much more sensitive to the $P T$ potential, as we discuss shortly.

The nucleon EDM contributions are found to be

$$
\begin{aligned}
\frac{1}{\sqrt{6}}\left\langle\Psi_{{ }^{3} \mathrm{He}}\left\|\vec{D}_{\not P P}^{(1)}\right\| \mid \Psi_{3_{\mathrm{He}}}\right\rangle & =0.88 d_{n}-0.047 d_{p}, \\
\frac{1}{\sqrt{6}}\left\langle\Psi_{{ }^{3} \mathrm{H}} \| \vec{D}_{P P T}^{(1)}|| \Psi_{{ }^{3} \mathrm{H}}\right\rangle & =-0.050 d_{n}+0.90 d_{p} .
\end{aligned}
$$

As expected, the helion (triton) EDM is mostly sensitive to the neutron (proton) EDM [42].

For the contribution from the PPT potential, our results for triton are very similar in magnitude to those for helion, in the case of OPE already obtained in Ref. [42]. The contribution of $\bar{C}_{1,2}$ as a function of $m_{1,2}$ is given in Fig. 4 for Argonne $v 18$. For each regulator mass, we perform calculations at four values of $\Omega=20,30,40,50 \mathrm{MeV}$, up to $N_{\max }=50$. We observe convergence and estimate a $10 \%$ error from the spread of results with $\Omega$. (See Fig. 1 of Ref. [42] for a generic convergence pattern.) As it can be seen from Fig. 4 , the results become approximately $m_{1,2}$ independent at large masses, implying that $\bar{C}_{1,2}$ approach constants in this limit. Results are very different for the 
EFT-inspired potential. Within the region of masses studied, we found an approximately linear dependence on the regulator mass, always larger in magnitude than for Argonne $v 18$. While for $m_{1}=m_{\omega}$ and $m_{2}=m_{\rho}$ the contributions to the tri-nucleon EDMs differ by a factor $\sim 2$ [42], the difference first increases and then decreases as $m_{1,2}$ increases, but it is still a factor of $\sim 5$ at $2.5 \mathrm{GeV}$. The linear regulator dependence could indicate a different running of $\bar{C}_{1,2}$, or simply a very slow convergence. However, calculations with this potential are computationally more intensive and we have been limited to $N_{\max }=40$, which increases the error. In any case, there is clearly a much stronger dependence of these short-range contributions on the potential, and more solid numbers have to await a fully consistent calculation. We quote here the Argonne $v 18$ numbers at $2.5 \mathrm{GeV}$, but we emphasize that they represent only an order of magnitude estimate. We obtain

$$
\begin{aligned}
\frac{2}{\sqrt{6}}\left\langle\Psi_{3_{\mathrm{He}}}\left\|\vec{D}_{P T}^{(1)}\right\| \widetilde{\Psi}_{3_{\mathrm{He}}}\right\rangle=\left(-0.15 \frac{\bar{g}_{0}}{F_{\pi}}-0.28 \frac{\bar{g}_{1}}{F_{\pi}}-0.01 F_{\pi}^{3} \bar{C}_{1}+0.02 F_{\pi}^{3} \bar{C}_{2}\right) \text { e fm }(.57) \\
\frac{2}{\sqrt{6}}\left\langle\Psi_{{ }^{\mathrm{H}}}\left\|\vec{D}_{P T}^{(1)}\right\| \widetilde{\Psi}_{3_{\mathrm{H}}}\right\rangle=\left(0.15 \frac{\bar{g}_{0}}{F_{\pi}}-0.28 \frac{\bar{g}_{1}}{F_{\pi}}+0.01 F_{\pi}^{3} \bar{C}_{1}-0.02 F_{\pi}^{3} \bar{C}_{2}\right) \text { e fm } .(58)
\end{aligned}
$$

In total, then, as anticipated in Sections 2.2 and 3 , the EDMs of helion and triton (as the EDMs of light nuclei in general) are functions of six PPT LECs:

$$
d_{3 \mathrm{He}}=0.88 d_{n}-0.047 d_{p}-\left(0.15 \frac{\bar{g}_{0}}{F_{\pi}}+0.28 \frac{\bar{g}_{1}}{F_{\pi}}+0.01 F_{\pi}^{3} \bar{C}_{1}-0.02 F_{\pi}^{3} \bar{C}_{2}\right) \text { efm }
$$

and

$$
d_{3_{\mathrm{H}}}=-0.050 d_{n}+0.90 d_{p}+\left(0.15 \frac{\bar{g}_{0}}{F_{\pi}}-0.28 \frac{\bar{g}_{1}}{F_{\pi}}+0.01 F_{\pi}^{3} \bar{C}_{1}-0.02 F_{\pi}^{3} \bar{C}_{2}\right) \text { efm }
$$

where $\bar{g}_{0}$ applies for $\bar{\theta}$, qCEDM, and $\chi \mathrm{I} ; \bar{g}_{1}$ for qCEDM and $\chi \mathrm{I} ; d_{n, p}$ for qEDM and $\chi \mathrm{I}$; and $\bar{C}_{1,2}$ for $\chi \mathrm{I}$ only.

Only in the case of the qEDM do we expect the tri-nucleon EDMs to be dominated by the nucleon EDMs. Not surprisingly, the helion (triton) EDM should be approximately equal to the neutron (proton) EDM. The nucleon EDM for the dimension-six sources was calculated in Ref. [26] and it was found that for qEDM the EDMs were dominated by the short-range contributions in Eqs. (21) and (22). In this case,

$$
\begin{aligned}
d_{{ }_{3} \mathrm{He}}(\mathrm{qEDM}) & =0.83 \bar{d}_{0}-0.93 \bar{d}_{1}, \\
d_{3_{\mathrm{H}}}(\mathrm{qEDM}) & =0.85 \bar{d}_{0}+0.95 \bar{d}_{1} .
\end{aligned}
$$

For the $\bar{\theta}$ term, on the other hand, the helion and triton EDMs depend at LO only on $\bar{g}_{0}$. To check this statement we compare the LO contribution with the contribution from the nucleon EDMs. If we assume the neutron and proton EDMs to be saturated by their long-range part, that is, the chiral log in Eq. (20), Eq. (24) shows that the short-range term is comparable to the pion-exchange contribution. To be on the safe side, 
it seems better not to neglect the LO nucleon EDMs for the $\bar{\theta}$ term, even though the power counting tells us it should be subleading; then

$$
\begin{aligned}
d_{3 \mathrm{He}}(\bar{\theta}) & =0.83 \bar{d}_{0}-0.93 \bar{d}_{1}-0.15 \frac{\bar{g}_{0}}{F_{\pi}} \text { efm } \\
d_{3{ }^{H} \mathrm{H}}(\bar{\theta}) & =0.85 \bar{d}_{0}+0.95 \bar{d}_{1}+0.15 \frac{\bar{g}_{0}}{F_{\pi}} \text { efm } .
\end{aligned}
$$

This argument holds equally well for the qCEDM, except that now also $\bar{g}_{1}$ contributes:

$$
\begin{aligned}
d_{3_{\mathrm{He}}}(\mathrm{qCEDM}) & =0.83 \bar{d}_{0}-0.93 \bar{d}_{1}-\left(0.15 \frac{\bar{g}_{0}}{F_{\pi}}+0.28 \frac{\bar{g}_{1}}{F_{\pi}}\right) e \mathrm{fm}, \\
d_{{ }_{3} \mathrm{H}}(\mathrm{qCEDM}) & =0.85 \bar{d}_{0}+0.95 \bar{d}_{1}+\left(0.15 \frac{\bar{g}_{0}}{F_{\pi}}-0.28 \frac{\bar{g}_{1}}{F_{\pi}}\right) e \mathrm{fm} .
\end{aligned}
$$

Finally in the case of $\chi \mathrm{I}$, we expect the tri-nucleon EDM to consist of $\bar{g}_{0,1}$ pion exchange, insertions of the PT short-range $N N$ interactions, and the contributions from the nucleon EDMs. Similarly to the qEDM, the nucleon EDM from $\chi \mathrm{I}$ is dominated by short-range contributions. All six LECs contribute:

$$
\begin{aligned}
d_{3 \mathrm{He}}(\chi \mathrm{I}) & =0.83 \bar{d}_{0}-0.93 \bar{d}_{1}-\left(0.15 \frac{\bar{g}_{0}}{F_{\pi}}+0.28 \frac{\bar{g}_{1}}{F_{\pi}}+0.01 F_{\pi}^{3} \bar{C}_{1}-0.02 F_{\pi}^{3} \bar{C}_{2}\right) \text { efm } \\
d_{3} \mathrm{H}(\chi \mathrm{I}) & =0.85 \bar{d}_{0}+0.95 \bar{d}_{1}+\left(0.15 \frac{\bar{g}_{0}}{F_{\pi}}-0.28 \frac{\bar{g}_{1}}{F_{\pi}}+0.01 F_{\pi}^{3} \bar{C}_{1}-0.02 F_{\pi}^{3} \bar{C}_{2}\right) \text { efm } .
\end{aligned}
$$

From Eq. 17) we infer $F_{\pi} \bar{d}_{0,1} / \bar{g}_{0}=\mathcal{O}\left(e F_{\pi} / m_{\pi}^{2}\right) \sim 2 e$ fm and $F_{\pi}^{4} \bar{C}_{1,2} / \bar{g}_{0}=\mathcal{O}\left(F_{\pi}^{2} / m_{\pi}^{2}\right) \sim$ 2. We see again that the $\bar{g}_{0,1}$ coefficients are somewhat smaller than expected; moreover, the PP short-range $N N$ interactions might contribute even less. However, one should keep in mind the large uncertainty in the $\bar{C}_{1,2}$ coefficients, and that these dimensional-analysis estimates could easily be offset by dimensionless factors in the LECs.

\section{Discussion and Conclusions}

Historically, hadronic EDMs have mostly been discussed in the framework of a one-bosonexchange model. It is assumed that $P$ - and $T$-violation is propagated by pions which are parametrized by three PPT non-derivative interactions. In our notation,

$$
\mathcal{L}=-\frac{\bar{g}_{0}}{F_{\pi}} \bar{N} \boldsymbol{\tau} \cdot \boldsymbol{\pi} N-\frac{\bar{g}_{1}}{F_{\pi}} \bar{N} \pi_{3} N-\frac{\bar{g}_{2}}{F_{\pi}} \bar{N} \tau_{3} \pi_{3} N
$$

(in the nuclear physics literature, where chiral symmetry and power counting are not emphasized, the coefficients are normally defined without $F_{\pi}$ ). Hadronic EDMs are calculated as a function of these three parameters. In some cases the effects of heavier bosons 
Table 1: Dependence of the EDMs of the neutron, proton, deuteron, helion, and triton on the six relevant PT low-energy constants. A "-" denotes that the LEC does not contribute in a model-independent way to the EDM at leading order. Values are for the Argonne $v 18$ potential; for the potential-model dependence of the results, see text.

\begin{tabular}{||c|cccccc||}
\hline LEC & $d_{0}$ & $d_{1}$ & $\left(\bar{g}_{0} / F_{\pi}\right) e f \mathrm{fm}$ & $\left(\bar{g}_{1} / F_{\pi}\right) e \mathrm{fm}$ & $\left(F_{\pi}^{3} \bar{C}_{1}\right) e \mathrm{fm}$ & $\left(F_{\pi}^{3} \bar{C}_{2}\right) e \mathrm{fm}$ \\
\hline$d_{n}$ & 1 & -1 & - & - & - & - \\
$d_{p}$ & 1 & 1 & - & - & - & - \\
$d_{2} \mathrm{H}$ & 2 & 0 & $0.0002-0.07 \beta_{1}$ & -0.19 & - & - \\
$d_{3} \mathrm{He}$ & 0.83 & -0.93 & -0.15 & -0.28 & -0.01 & 0.02 \\
$d_{3} \mathrm{H}$ & 0.85 & 0.95 & 0.15 & -0.28 & 0.01 & -0.02 \\
\hline
\end{tabular}

are included as well. In this work we argue that this model is oversimplified. There is a priori no reason not to include PPT photon-nucleon and short-range nucleon-nucleon interactions at low energies. By studying the chiral properties of the fundamental PPT sources of dimension up to six at the QCD scale, it is possible to construct a modelindependent hadronic PF Lagrangian with a definite hierarchy between the different PT hadronic interactions. It is found that the one-pion-exchange model with three LECs is not appropriate for any of these PP sources, and in general there are six PF hadronic interactions that determine the EDMs of light nuclei. Two of those are in the OPE model as well $-\bar{g}_{0}$ and $\bar{g}_{1}$ - and the other four are additional interactions that need to be considered when determining hadronic EDMs. The $\bar{g}_{2}$ interaction is not relevant at LO for any of the fundamental sources. The other four necessary LECs are the isoscalar and isovector components of the neutron and proton EDMs and two isoscalar PPT NN interactions of short range. The isovector PP $N N$ interactions come in at higher order for all sources.

We therefore propose that nuclear EDMs be analyzed on the basis of these six LECs. In the previous sections we discussed EDMs of light nuclei, providing specific examples in the form of the deuteron, helion, and triton. In Table 1 the dependence of these various EDMs on the six LECs is summarized. From the table it is clear that using the OPE model gives an oversimplified view. At least six observables are required to identify the six LECs. If other light nuclei become the target of experimental investigation, their EDMs can be calculated along similar lines at the cost of larger computer resources. We hope that EDMs of heavier systems can be also expressed in terms of these six LECs. However, in these cases there could be significant enhancement factors for the PT potential contribution [50], making important otherwise subleading terms in the potential [34], such as the third non-derivative pion-nucleon coupling $\bar{g}_{2}$.

Once (a subset of) the LECs are determined it is possible to learn something about the more fundamental PPT sources at the QCD scale. In Table 2 we list for the different PPT sources the expected orders of magnitude of the neutron EDM, $d_{n}$, and ratios between the other EDMs considered here and $d_{n}$. Although some care is needed when using this table - as we have discussed, the numbers found earlier are not always exactly of the expected 
Table 2: Expected orders of magnitude for the neutron EDM (in units of $e / M_{\mathrm{QCD}}$ ), the ratio of proton-to-neutron EDMs, the ratio of deuteron-to-neutron EDMs, the ratio of helion-to-neutron EDMs, and the ratio of triton-to-neutron EDMs, for the $\bar{\theta}$ term and the three dimension-six sources. $Q$ stands for the low-energy scales $F_{\pi}, m_{\pi}$, and $\gamma$.

\begin{tabular}{||c|cccc||}
\hline Source & $\bar{\theta}$ & $\mathrm{qCEDM}$ & $\mathrm{qEDM}$ & $\chi \mathrm{I}$ \\
\hline$M_{\mathrm{QCD}} d_{n} / e$ & $\mathcal{O}\left(\bar{\theta} \frac{m_{\pi}^{2}}{M_{\mathrm{QCD}}^{2}}\right)$ & $\mathcal{O}\left(\tilde{\delta} \frac{m_{\pi}^{2}}{M_{T}^{2}}\right)$ & $\mathcal{O}\left(\delta \frac{m_{\pi}^{2}}{M_{T}^{2}}\right)$ & $\mathcal{O}\left(w \frac{M_{\mathrm{QCD}}^{2}}{M_{T}^{2}}\right)$ \\
$d_{p} / d_{n}$ & $\mathcal{O}(1)$ & $\mathcal{O}(1)$ & $\mathcal{O}(1)$ & $\mathcal{O}(1)$ \\
$d_{{ } \mathrm{H}} / d_{n}$ & $\mathcal{O}(1)$ & $\mathcal{O}\left(\frac{M_{\mathrm{QCD}}^{2}}{Q^{2}}\right)$ & $\mathcal{O}(1)$ & $\mathcal{O}(1)$ \\
$d_{3} \mathrm{He} / d_{n}$ & $\mathcal{O}\left(\frac{M_{\mathrm{QCD}}^{2}}{Q^{2}}\right)$ & $\mathcal{O}\left(\frac{M_{\mathrm{QCD}}^{2}}{Q^{2}}\right)$ & $\mathcal{O}(1)$ & $\mathcal{O}(1)$ \\
$d_{{ }_{3} \mathrm{H}} / d_{n}$ & $\mathcal{O}\left(\frac{M_{\mathrm{QCD}}^{2}}{Q^{2}}\right)$ & $\mathcal{O}\left(\frac{M_{\mathrm{QCD}}^{2}}{Q^{2}}\right)$ & $\mathcal{O}(1)$ & $\mathcal{O}(1)$ \\
\hline
\end{tabular}

size - it does allow some qualitative statements, even if less than six measurements are available.

The simplest scenario is the one where PT is dominated by the qEDM, in which case all light nuclear EDMs are essentially given by two LECs only: $\bar{d}_{0}$ and $\bar{d}_{1}$. (See Eqs. (21), (22), (50), 61), and (62).) A measurement of the proton and neutron EDMs would make deuteron and tri-nucleon EDMs testable predictions,

$$
\begin{aligned}
d_{2} \mathrm{H} & \simeq d_{n}+d_{p}, \\
d_{{ }^{3} \mathrm{He}}+d_{3^{3} \mathrm{H}} & \simeq 0.84\left(d_{n}+d_{p}\right), \\
d_{{ }^{3} \mathrm{He}}-d_{3^{\mathrm{H}}} & \simeq 0.94\left(d_{n}-d_{p}\right) .
\end{aligned}
$$

The nucleon Schiff moments and the deuteron magnetic quadrupole moment (MQM) depend on other LECs [26, 28] and cannot be predicted. For light nuclei the effects of the PT potential from the qEDM are suppressed compared to the nucleon EDMs [34], although enhancements could make them more relevant for heavier nuclei.

PT from $\bar{\theta}$ and qCEDM manifests itself in EDMs of light nuclei that differ significantly from the EDMs of their constituents. For both sources, the EDMs we calculated depend at LO on four of the six LECs $-\bar{g}_{0}, \bar{g}_{1}, \bar{d}_{0}$, and $\bar{d}_{1}-$ but in different ways. For the qCEDM, the distinguishing feature is that the deuteron EDM (49) is expected to be significantly larger than the isoscalar nucleon EDM, thanks to $\bar{g}_{1}$. Thus, a measurement of nucleon and deuteron EDMs could be sufficient to qualitatively pinpoint, or exclude, qCEDM as a dominant PPT source, and to fix the values of $\bar{d}_{0,1}$ and $\bar{g}_{1}$. Then, the isoscalar combination of helion and triton EDMs, $d_{{ }^{3} \mathrm{He}}+d_{3_{3} \mathrm{H}}$, which in LO only depends on $\bar{g}_{1}$, becomes a falsifiable prediction of the theory,

$$
d^{3} \mathrm{He}+d_{3} \mathrm{H} \simeq 3 d_{2} \mathrm{H} .
$$

If, to be on the safe side, we keep some subleading terms (the nucleon EDMs) as we did in Eqs. (65) and (66), then we get (including the subleading term $2 \bar{d}_{0}$ in Eq. (49)) an 
additional $-2.16\left(d_{n}+d_{p}\right)$ on the right-hand side of Eq. $(73)$. Furthermore, $\bar{g}_{0}$ can then be extracted from $d_{3} \mathrm{He}-d_{3} \mathrm{H}$ (see Eqs. (65) and (66)), leading to testable predictions for other PP observables.

In contrast, for the Standard Model $\bar{\theta}$ term we do not expect the deuteron EDM to be significantly different from twice the isoscalar nucleon EDM. Although the deuteron EDM (52) formally depends on the isoscalar nucleon EDM and on the pion-nucleon couplings $\bar{g}_{0}$ and $\bar{g}_{1}$, the results of Section 5 show that the pion-exchange contribution is likely only $\sim 10 \%$ of the nucleon EDM. On the other hand, the EDMs of ${ }^{3} \mathrm{He}$ and ${ }^{3} \mathrm{H}$, Eqs. 63. and (64), are dominated by $\bar{g}_{0}$, although they receive important contributions from the neutron and proton EDMs. In particular, we expect the isovector combination $d_{3 \mathrm{He}}-d_{3_{3} \mathrm{H}}$, which is sensitive to $\bar{g}_{0}$, to differ from the isovector nucleon EDM $\bar{d}_{1}$, while the isoscalar combination $d_{3} \mathrm{He}+d_{3} \mathrm{H}$ should be close to $2 \bar{d}_{0}$ :

$$
\begin{aligned}
d^{3^{\mathrm{He}}}+d_{3 \mathrm{H}} & \simeq 0.84\left(d_{n}+d_{p}\right), \\
d_{3^{3} \mathrm{He}}-d_{3^{3} \mathrm{H}} & \neq 0.94\left(d_{n}-d_{p}\right) .
\end{aligned}
$$

The experimental observation of these relations in nucleon, deuteron, helion, and triton EDM experiments would qualitatively indicate the $\bar{\theta}$ term as the main source responsible for PT. Quantitatively, the measurement of nucleon, helion, and triton EDM allows extraction of the coupling $\bar{g}_{0}$, which then can be used to provide testable predictions of other PP observables, like the proton Schiff moment [26, 20] or the deuteron MQM [28], which are not sensitive to the nucleon EDMs.

Finally, in the case of the $\chi \mathrm{I}$ sources the analysis is in principle most complicated, due to the appearance of all six LECs. Like for $\bar{\theta}$, the deuteron EDM (51), although formally dependent on $\bar{g}_{1}$ at LO, is probably dominated by $\bar{d}_{0}$. The tri-nucleon EDMs (67) and (68) formally depend on all six LECs, but they are again possibly dominated by $d_{0}$ and $d_{1}$. It might thus be difficult to separate the $\chi \mathrm{I}$ sources from qEDM. For less dilute, but still light, systems we expect different results. For these systems, in the case of qEDM the EDMs are still dominated by $\bar{d}_{0,1}$, but for $\chi \mathrm{I}$ sources we expect the contributions from the PF potential to be more significant, implying that measurements on these systems might separate $\chi \mathrm{I}$ sources from qEDM. Of course, more extensive calculations are necessary to verify this claim.

In conclusion, we have argued that an experimental program to measure light nuclear EDMs could offer valuable information on yet undiscovered sources of parity and timereversal violation. Our case is based on some crucial, but relatively general assumptions, such as the validity of the Standard Model with its minimal particle content at the electroweak scale, and the naturalness of interaction strengths. Elsewhere [28] it has already been pointed out - basically on the basis of dimensional analysis - that sensitivity to the deuteron EDM at the level hoped for in storage ring experiments [6] would probe scales where new physics is expected. A similar analysis holds for our tri-nucleon results, Eqs. (59) and (60). But our results here go beyond dimensional analysis and suggest that, at least for the lightest nuclei, the contribution of the neutron and proton EDMs are more important than expected by simple power counting. For all sources, they compete with, when they do not dominate, the effects of the PT potential. For this reason, other PT 
observables insensitive to the nucleon EDMs, for example higher PT electromagnetic moments, could provide important complementary information and a cleaner way to extract pion-nucleon and nucleon-nucleon PT couplings. Additionally, it would be interesting if EDMs of heavier systems could be recast in terms of our EFT approach.

\section{Acknowledgments}

We thank the organizers of the Workshop on Search for Electric Dipole Moments at Storage Rings (Physikzentrum Bad Honnef, July 2011), H. Ströher and F. Rathmann, for providing a stimulating atmosphere for discussions with many colleagues, in particular J. Bsaisou, B. Gibson, C. Hanhart, A. Nogga, G. Onderwater, and A. Wirzba. We are particularly grateful to P. Navrátil for the NCSM code of the PT wavefunctions, and to C. Hanhart for comments on the manuscript. C.-P. Liu and U. van Kolck acknowledge the hospitality of KVI, where this research was carried out. This research was supported by the Dutch Stichting FOM under programs 104 and 114 (JdV, RH, RGET), by the ROC NSC under grant NSC98-2112-M-259-004-MY3 (CPL), and by the US DOE under grants DE-FG02-06ER41449 (EM), DE-FG02-04ER41338 (EM, UvK), and DEFC02-07ER41457 (IS). The three-body calculations were performed on the University of Washington Hyak cluster (NSF MRI grant PHY-0922770).

\section{A Potential in Coordinate Space}

In configuration space, the LO potential of Sect. 3.2 is given by [34]

$$
\begin{aligned}
V_{\text {PPF }}(\vec{r})= & -\frac{\bar{g}_{0} g_{A}}{F_{\pi}^{2}} \boldsymbol{\tau}^{(i)} \cdot \boldsymbol{\tau}^{(j)}\left(\vec{\sigma}^{(i)}-\vec{\sigma}^{(j)}\right) \cdot\left(\vec{\nabla}_{r} U(r)\right) \\
& -\frac{\bar{g}_{1} g_{A}}{2 F_{\pi}^{2}}\left[\left(\tau_{3}^{(i)}+\tau_{3}^{(j)}\right)\left(\vec{\sigma}^{(i)}-\vec{\sigma}^{(j)}\right)+\left(\tau_{3}^{(i)}-\tau_{3}^{(j)}\right)\left(\vec{\sigma}^{(i)}+\vec{\sigma}^{(j)}\right)\right] \cdot\left(\vec{\nabla}_{r} U(r)\right) \\
& +\frac{1}{2}\left[\bar{C}_{1}+\bar{C}_{2} \boldsymbol{\tau}^{(i)} \cdot \boldsymbol{\tau}^{(j)}\right]\left(\vec{\sigma}^{(i)}-\vec{\sigma}^{(j)}\right) \cdot\left(\vec{\nabla}_{r} \delta^{(3)}(\vec{r})\right)
\end{aligned}
$$

where $\vec{r}=\vec{x}_{i}-\vec{x}_{j}$ is the relative position of the two interacting nucleons and

$$
U(r)=\frac{1}{12 \pi r}\left[2 \exp \left(-m_{\pi^{ \pm}} r\right)+\exp \left(-m_{\pi^{0}} r\right)\right]
$$

which reduces to the usual Yukawa function $U(r)=\exp \left(-m_{\pi} r\right) / 4 \pi r$ when, at LO, we ignore the pion mass difference. 
Analogously, the NNLO potential of Sect. 4.2 becomes 34

$$
\begin{aligned}
V_{\text {PPF }}\left(\vec{r}, \vec{\nabla}_{r}, \vec{\nabla}_{X}\right)= & -\frac{\bar{g}_{0} g_{A}}{2 F_{\pi}^{2}}\left[\left(\frac{\bar{g}_{1}}{\bar{g}_{0}}-\frac{\beta_{1}}{2 g_{A}}\right)\left(\tau_{3}^{(i)}+\tau_{3}^{(j)}\right)\left(\vec{\sigma}^{(i)}-\vec{\sigma}^{(j)}\right)\right. \\
& \left.+\left(\frac{\bar{g}_{1}}{\bar{g}_{0}}+\frac{\beta_{1}}{2 g_{A}}\right)\left(\tau_{3}^{(i)}-\tau_{3}^{(j)}\right)\left(\vec{\sigma}^{(i)}+\vec{\sigma}^{(j)}\right)\right] \cdot\left(\vec{\nabla}_{r} U(r)\right) \\
& +\frac{\bar{g}_{0} g_{A}}{3 F_{\pi}^{2}}\left(3 \tau_{3}^{(i)} \tau_{3}^{(j)}-\tau^{(i)} \cdot \boldsymbol{\tau}^{(j)}\right) \\
& \times\left(\vec{\sigma}^{(i)}-\vec{\sigma}^{(j)}\right) \cdot\left[\frac{\delta m_{N}^{2}}{2 m_{\pi}}\left(\vec{\nabla}_{r} r U(r)\right)+\left(\vec{\nabla}_{r} W(r)\right)\right] \\
& -i \frac{\bar{g}_{0} g_{A}}{2 F_{\pi}^{2}} \frac{\delta m_{N}}{m_{N}}\left(\tau^{(i)} \times \tau^{(j)}\right)_{3}\left\{\left(\vec{\sigma}^{(i)}+\vec{\sigma}^{(j)}\right) \cdot\left\{\vec{\nabla}_{r}, U(r)\right\}\right. \\
& \left.+\left(\vec{\sigma}^{(i)}-\vec{\sigma}^{(j)}\right) \cdot\left[U(r) \vec{\nabla}_{X}-\frac{1}{m_{\pi}}\left(\vec{\nabla}_{r} \nabla_{r}^{n} r U(r)\right) \nabla_{X}^{n}\right]\right\},
\end{aligned}
$$

where $\vec{X}=\left(\vec{x}_{i}+\vec{x}_{j}\right) / 2$ and

$$
W(r)=\frac{1}{4 \pi r}\left[\exp \left(-m_{\pi^{ \pm}} r\right)-\exp \left(-m_{\pi^{0}} r\right)\right]
$$

which is entirely a consequence of isospin breaking.

\section{B Fourier Transform of the Currents}

To evaluate the matrix elements in Section 5 we need to transform the currents to configuration space. We follow Ref. [64] and transform with respect to the nucleon momenta but not with respect to the photon momentum. In the most general case

$$
\begin{aligned}
J^{0}\left(\vec{x}_{i}, \vec{x}_{i}^{\prime}, \vec{x}_{j}, \vec{x}_{j}^{\prime}, \vec{q}\right)= & \int \frac{\mathrm{d}^{3} p_{i}}{(2 \pi)^{3}} \int \frac{\mathrm{d}^{3} p_{i}^{\prime}}{(2 \pi)^{3}} \int \frac{\mathrm{d}^{3} p_{j}}{(2 \pi)^{3}} \int \frac{\mathrm{d}^{3} p_{j}^{\prime}}{(2 \pi)^{3}} e^{-i \vec{p}_{i} \cdot \vec{x}_{i}} e^{-i \vec{p}_{j} \cdot \vec{x}_{j}} e^{i \vec{p}_{i}^{\prime} \cdot \vec{x}_{i}^{\prime}} e^{i \vec{p}_{j}^{\prime} \cdot \vec{x}_{j}^{\prime}} \\
& (2 \pi)^{3} \delta^{(3)}\left(\vec{p}_{i}+\vec{p}_{j}-\vec{p}_{i}^{\prime}-\vec{p}_{j}^{\prime}-\vec{q}\right) J^{0}\left(\vec{p}_{i}, \vec{p}_{i}^{\prime}, \vec{p}_{j}, \vec{p}_{j}^{\prime}, \vec{q}\right) .
\end{aligned}
$$

Introducing the relative configuration-space coordinates $\vec{r}=\vec{x}_{i}-\vec{x}_{j}, \vec{r}^{\prime}=\vec{x}_{i}^{\prime}-\vec{x}_{j}^{\prime}, \vec{X}=$ $\left(\vec{x}_{i}+\vec{x}_{j}\right) / 2$, and $\vec{X}^{\prime}=\left(\vec{x}_{i}^{\prime}+\vec{x}_{j}^{\prime}\right) / 2$, we rewrite this as

$$
\begin{aligned}
& J^{0}\left(\vec{r}, \vec{r}^{\prime}, \vec{X}, \vec{X}^{\prime}, \vec{q}\right)=e^{-\frac{i}{2} \vec{q} \cdot\left(\vec{X}+\vec{X}^{\prime}\right)} \int \frac{\mathrm{d}^{3} P_{t}}{(2 \pi)^{3}} \int \frac{\mathrm{d}^{3} K}{(2 \pi)^{3}} \int \frac{\mathrm{d}^{3} k}{(2 \pi)^{3}} \\
& e^{-i \vec{P}_{t} \cdot\left(\vec{X}-\vec{X}^{\prime}\right)} e^{-i \vec{K} \cdot\left(\vec{r}-\vec{r}^{\prime}\right)} e^{-\frac{i}{2} \vec{k} \cdot\left(\vec{r}+\vec{r}^{\prime}\right)} J^{0}\left(\vec{q}, \vec{k}, \vec{K}, \vec{P}_{t}\right) .
\end{aligned}
$$

The currents we need (the third currents in Eqs. 36 and $(38)$ ) depend on $\vec{q}$ and $\vec{k}$ only, such that the expression can be simplified to

$$
J^{0}(\vec{r}, \vec{X}, \vec{q})=e^{-i \vec{q} \cdot \vec{X}} \int \frac{\mathrm{d}^{3} k}{(2 \pi)^{3}} e^{-i \vec{k} \cdot \vec{r}} J^{0}(\vec{q}, \vec{k}) .
$$


The Fourier transforms can be done and we find for the required currents

$$
\begin{aligned}
J_{P T, c}^{0}(\vec{r}, \vec{X}, \vec{q})= & -\frac{2 e g_{A}^{2}}{F_{\pi}^{2}} \delta m_{N}\left(\boldsymbol{\tau}^{(i)} \cdot \boldsymbol{\tau}^{(j)}-\tau_{3}^{(i)} \tau_{3}^{(j)}\right) \\
& \times e^{-i \vec{q} \cdot \vec{X}}\left[\vec{\sigma}^{(i)} \cdot\left(i \vec{\nabla}_{r}+\frac{\vec{q}}{2}\right) \vec{\sigma}^{(j)} \cdot\left(i \vec{\nabla}_{r}-\frac{\vec{q}}{2}\right)\right] W(\vec{q}, \vec{r}), \\
J_{P P, c}^{0}(\vec{r}, \vec{X}, \vec{q})= & \frac{2 i e g_{A} \bar{g}_{0}}{F_{\pi}^{2}} \delta m_{N}\left(\boldsymbol{\tau}^{(i)} \cdot \boldsymbol{\tau}^{(j)}-\tau_{3}^{(i)} \tau_{3}^{(j)}\right) \\
& \times e^{-i \vec{q} \cdot \vec{X}}\left[\left(\vec{\sigma}^{(i)}+\vec{\sigma}^{(j)}\right) \cdot \frac{\vec{q}}{2}+\left(\vec{\sigma}^{(i)}-\vec{\sigma}^{(j)}\right) \cdot\left(i \vec{\nabla}_{r}\right)\right] W(\vec{q}, \vec{r}),
\end{aligned}
$$

in terms of the function

$$
W(\vec{q}, \vec{r})=\frac{1}{8 \pi} \int_{0}^{1} \mathrm{~d} \alpha \exp \left[i \frac{\vec{q} \cdot \vec{r}}{2}(1-2 \alpha)\right] \frac{\exp \left[-r\left(m_{\pi}^{2}+\vec{q}^{2} \alpha(1-\alpha)\right)^{1 / 2}\right]}{\left[m_{\pi}^{2}+\vec{q}^{2} \alpha(1-\alpha)\right]^{1 / 2}} .
$$

Before continuing it is convenient to look at the inverse Fourier transform of the current

$$
\begin{aligned}
J^{0}(\vec{q}) & =\int \mathrm{d}^{3} x e^{-i \vec{q} \cdot \vec{x}} J^{0}(\vec{x}) \\
& =\int \mathrm{d}^{3} x J^{0}(\vec{x})-i \vec{q} \cdot \int \mathrm{d}^{3} x \vec{x} J^{0}(\vec{x})+\mathcal{O}\left(\vec{q}^{2}\right) \\
& =Z e-i \vec{q} \cdot \vec{D}+\mathcal{O}\left(\vec{q}^{2}\right),
\end{aligned}
$$

where $Z e$ is the total charge and $\vec{D}$ is the EDM operator used in Sections 5 and 6 . An easy way to extract the EDM operator is by using

$$
\vec{D}=i \lim _{q \rightarrow 0} \vec{\nabla}_{q} J^{0}(\vec{q}) .
$$

As an example we consider the EDM operator coming from $J_{P \mathcal{P}, c}^{0}(\vec{r}, \vec{X}, \vec{q})$. From Eq. (87) we read off

$$
\begin{aligned}
& \vec{D}_{\not P T, c}=\frac{2 i e g_{A} \bar{g}_{0}}{F_{\pi}^{2}} \delta m_{N}\left(\boldsymbol{\tau}^{(i)} \cdot \boldsymbol{\tau}^{(j)}-\tau_{3}^{(i)} \tau_{3}^{(j)}\right) \\
& \times\left[\frac{i}{2}\left(\vec{\sigma}^{(i)}+\vec{\sigma}^{(j)}\right)+\vec{X}\left(\vec{\sigma}^{(i)}-\vec{\sigma}^{(j)}\right) \cdot\left(i \vec{\nabla}_{r}\right)\right] \frac{e^{-m_{\pi} r}}{8 \pi m_{\pi}} \\
& =-\frac{e g_{A} \bar{g}_{0}}{F_{\pi}^{2}} \delta m_{N}\left(\boldsymbol{\tau}^{(i)} \cdot \boldsymbol{\tau}^{(j)}-\tau_{3}^{(i)} \tau_{3}^{(j)}\right) \\
& \times\left[\left(\vec{\sigma}^{(i)} \cdot \vec{\nabla}^{(i)}+\vec{\sigma}^{(j)} \cdot \vec{\nabla}^{(j)}\right)\left(\vec{x}_{i}+\vec{x}_{j}\right) \frac{e^{-m_{\pi}\left|\vec{x}_{i}-\vec{x}_{j}\right|}}{8 \pi m_{\pi}}\right],
\end{aligned}
$$

where we used $\lim _{q \rightarrow 0} \vec{\nabla}_{q} W(\vec{q}, \vec{r})=0$. This is Eq. (43). Following similar steps we obtain Eq. (45) from $J_{P T, c}^{0}(\vec{r}, \vec{X}, \vec{q})$. 


\section{References}

[1] I. B. Khriplovich and S. K. Lamoreaux, CP Violation Without Strangeness: Electric Dipole Moments of Particles, Atoms, and Molecules (Springer Verlag, Berlin, 1997).

[2] M. Pospelov and A. Ritz, Ann. Phys. 318, 119 (2005).

[3] M. Kobayashi and T. Maskawa, Prog. Theor. Phys. 49, 652 (1973).

[4] T. M. Ito, J. Phys. Conf. Ser. 69, 012037 (2007), nucl-ex/0702024; K. Bodek et al., arXiv:0806.4837 [nucl-ex].

[5] C. A. Baker et al., Phys. Rev. Lett. 97, 131801 (2006).

[6] F. J. M. Farley et al., Phys. Rev. Lett. 93, 052001 (2004); Y. F. Orlov, W. M. Morse, and Y. K. Semertzidis, Phys. Rev. Lett. 96, 214802 (2006); C. J. G. Onderwater, J. Phys. Conf. Ser. 295, 012008 (2011).

[7] W. C. Griffith et al., Phys. Rev. Lett. 102, 101601 (2009).

[8] I. B. Khriplovich and A. R. Zhitnitsky, Phys. Lett. B 109, 490 (1982); B. H. J. McKellar, S. R. Choudhury, X.-G. He, and S. Pakvasa, Phys. Lett. B 197, 556 (1987); X.-G. He, B. H. J. McKellar, and S. Pakvasa, Int. J. Mod. Phys. A4, 5011 (1989); A6, 1063(E) (1991); M. E. Pospelov, Phys. Lett. B 328, 441 (1994); A. Czarnecki and B. Krause, Phys. Rev. Lett. 78, 4339 (1997).

[9] G. 't Hooft, Phys. Rev. Lett. 37, 8 (1976); C. G. Callan, Jr, R. F. Dashen, and D. J. Gross, Phys. Lett. B 63, 334 (1976); R. Jackiw and C. Rebbi, Phys. Rev. Lett. 37, 172 (1976).

[10] V. Baluni, Phys. Rev. D 19, 2227 (1979).

[11] R. J. Crewther, P. Di Vecchia, G. Veneziano, and E. Witten, Phys. Lett. B 88, 123 (1979); 91, 487(E) (1980).

[12] W. Buchmüller and D. Wyler, Nucl. Phys. B268, 621 (1986); A. De Rújula, M. B. Gavela, O. Pène, and F. J. Vegas, Nucl. Phys. B357, 311 (1991).

[13] S. Weinberg, Phys. Rev. Lett. 63, 2333 (1989).

[14] B. Grzadkowski, M. Iskrzynski, M. Misiak, and J. Rosiek, JHEP 1010, 085 (2010).

[15] M. J. Ramsey-Musolf and S. Su, Phys. Rept. 456, 1 (2008).

[16] S. Weinberg, Physica 96A, 327 (1979); J. Gasser and H. Leutwyler, Ann. Phys. 158, 142 (1984); Nucl. Phys. B250, 465 (1985).

[17] E. Jenkins and A. V. Manohar, Phys. Lett. B 255, 558 (1991). 
[18] S. Weinberg, The Quantum Theory of Fields, Vol. 2 (Cambridge University Press, Cambridge, 1996).

[19] V. Bernard, N. Kaiser, and U.-G Meißner, Int. J. Mod. Phys. E 4, 193 (1995).

[20] S. D. Thomas, Phys. Rev. D 51, 3955 (1995).

[21] W. H. Hockings and U. van Kolck, Phys. Lett. B 605, 273 (2005).

[22] E. Mereghetti, W. H. Hockings, and U. van Kolck, Ann. Phys. 325, 2363 (2010).

[23] E. Mereghetti, J. de Vries, W. H. Hockings, C. M. Maekawa, and U. van Kolck, Phys. Lett. B 696, 97 (2011).

[24] H.-Y. Cheng, Phys. Rev. D 44, 166 (1991); A. Pich and E. de Rafael, Nucl. Phys. B367, 313 (1991); P. Cho, Phys. Rev. D 48, 3304 (1993); B. Borasoy, Phys. Rev. D 61, 114017 (2000).

[25] S. Narison, Phys. Lett. B 666, 455 (2008); K. Ottnad, B. Kubis, U.-G. Meißner, and F.-K. Guo, Phys. Lett. B 687, 42 (2010).

[26] J. de Vries, E. Mereghetti, R. G. E. Timmermans, and U. van Kolck, Phys. Lett. B 695, 268 (2011).

[27] J. de Vries, E. Mereghetti, R. G. E. Timmermans, and U. van Kolck, in preparation.

[28] J. de Vries, E. Mereghetti, R.G.E. Timmermans, and U. van Kolck, Phys. Rev. Lett. 107, 091804 (2011).

[29] D. B. Kaplan, M. J. Savage, and M. B. Wise, Nucl. Phys. B534, 329 (1998); S. Fleming, T. Mehen, and I.W. Stewart, Nucl. Phys. A677, 313 (2000).

[30] P. F. Bedaque and U. van Kolck, Ann. Rev. Nucl. Part. Sci. 52, 339 (2002);

[31] S. Weinberg, Nucl. Phys. B 363, 3 (1991).

[32] U. van Kolck, Ph.D. dissertation, University of Texas (1993); Few-Body Syst. Suppl. 9, 444 (1995).

[33] S. R. Beane, P. F. Bedaque, M. J. Savage, and U. van Kolck, Nucl. Phys. A 700, 377 (2002); A. Nogga, R. G. E. Timmermans, and U. van Kolck, Phys. Rev. C 72, 054006 (2005); M. C. Birse, Phys. Rev. C 74, 014003 (2006); 76, 034002 (2007); M. Pavón Valderrama, Phys. Rev. C 83, 024003 (2011); arXiv:1108.0872 [nucl-th]; Bingwei Long and C.-J. Yang, arXiv:1108.0985 [nucl-th].

[34] C. M. Maekawa, E. Mereghetti, J. de Vries, and U. van Kolck, arXiv:1106.6119 [nucl-th]. 
[35] J. Bsaisou, C. Hanhart, S. Liebig, A. Nogga, and A. Wirzba, talk at the Workshop on Search for Electric Dipole Moments at Storage Rings, Physikzentrum Bad Honnef, July 2011, www2.fz-juelich.de/ikp/edm/en/abstract.php?IDABS=28.

[36] O. P. Sushkov, V. V. Flambaum, and I. B. Khriplovich, Sov. Phys. JETP 60, 873 (1984).

[37] Y. Avishai, Phys. Rev. D 32, 314 (1985).

[38] I. B. Khriplovich and R. V. Korkin, Nucl. Phys. A665, 365 (2000).

[39] C.-P. Liu and R. G. E. Timmermans, Phys. Rev. C 70, 055501 (2004).

[40] I. R. Afnan and B. F. Gibson, Phys. Rev. C 82, 064002 (2010).

[41] Y. Avishai and M. Fabre de la Ripelle, Phys. Rev. Lett. 56, 2121 (1986); Nucl. Phys. A468, 578 (1987).

[42] I. Stetcu, C.-P. Liu, J. L. Friar, A. C. Hayes, and P. Navrátil, Phys. Lett. B 665, 168 (2008).

[43] P. Herczeg, Nucl. Phys. 75, 655 (1966); C.-P. Liu and R. G. E. Timmermans, Phys. Lett. B 634, 488 (2006).

[44] V. G. J. Stoks, R. A. M. Klomp, C. P. F. Terheggen, and J. J. de Swart, Phys. Rev. C 49, 2950 (1994).

[45] R. B. Wiringa, V. G. J. Stoks, and R. Schiavilla, Phys. Rev. C 51, 38 (1995).

[46] D. R. Entem and R. Machleidt, Phys. Rev. C 68, 041001(R) (2003); E. Epelbaum, A. Nogga, W. Glöckle, H. Kamada, U.G. Meißner, and H. Witała, Phys. Rev. C 66, 064001 (2002).

[47] S. Weinberg, Phys. Lett. B 295, 114 (1992).

[48] D. R. Phillips, Phys. Lett. B 567, 12 (2003).

[49] C.-P. Liu, Phys. Rev. C 75, 065501 (2007).

[50] W.C. Haxton and E.M. Henley, Phys. Rev. Lett. 51, 1937 (1983).

[51] A. Manohar and H. Georgi, Nucl. Phys. B234, 189 (1984).

[52] V. Bernard, N. Kaiser, J. Kambor, and U.-G. Meißner, Nucl. Phys. B388, 315 (1992);

N. Fettes, U.-G. Meißner, and S. Steininger, Nucl. Phys. A640, 199 (1998).

[53] J. L. Friar, U. van Kolck, M. C. M. Rentmeester, and R. G. E. Timmermans, Phys. Rev. C 70, 044001 (2004). 
[54] K. Nakamura [Particle Data Group], J. Phys. G 37, 075021 (2010).

[55] S. R. Beane, K. Orginos, and M. J. Savage, Nucl. Phys. B768, 38 (2007).

[56] U. van Kolck, J. A. Niskanen, and G. A. Miller, Phys. Lett. B 493, 65 (2000); D. R. Bolton and G. A. Miller, Phys. Rev. C 81, 014001 (2010); A. Filin et al., Phys. Lett. B 681, 423 (2009).

[57] J. Gasser and H. Leutwyler, Phys. Rep. 87, 77 (1982).

[58] V. G. J. Stoks, R. Timmermans, and J. J. de Swart, Phys. Rev. C 47, 512 (1993); M. C. M. Rentmeester, R. G. E. Timmermans, J.L. Friar, and J. J. de Swart, Phys. Rev. Lett. 82, 4992 (1999).

[59] U. van Kolck, J. L. Friar, and T. Goldman, Phys. Lett. B 371, 169 (1996); U. van Kolck, M. C. M. Rentmeester, J. L. Friar, T. Goldman, and J. J. de Swart, Phys. Rev. Lett. 80, 4386 (1998).

[60] A. V. Manohar, Phys. Rev. D 56, 230 (1997).

[61] S. Liebig, V. Baru, F. Ballout, C. Hanhart, A. Nogga, Eur. Phys. J. A47, 69 (2011).

[62] P. Navrátil, J. P. Vary, and B. R. Barrett, Phys. Rev. Lett. 84, 5728 (2000); Phys. Rev. C 62, 054311 (2000).

[63] P. Navrátil, G. Kamuntavicius, and B. R. Barrett, Phys. Rev. C 61, 044001 (2000).

[64] S. Kölling, E. Epelbaum, H. Krebs, and U.-G. Meißner, arXiv:1107.0602 [nucl-th]. 